\title{
La competencia aprender a aprender: valoración de un modelo teórico
}

\author{
The learning to learn competence: \\ An assessment of a theoretical model
}

\begin{abstract}
Dr. Bernardo GARGALLO LÓPEZ. Catedrático. Universidad de Valencia (bernardo.gargallo@uv.es).
Fran J. GARCÍA-GARCÍA. Personal Investigador en Formación. Universidad de Valencia (Francisco.Javier.Garcia-Garcia@uv.es). Dra. Inmaculada LÓPEZ-FRANCÉS. Ayudante Doctora. Universidad de Valencia (Inmaculada.Lopez-Frances@uv.es). Dr. Miguel Ángel JIMÉNEZ RODRÍGUEZ. Acreditado Doctor. Universidad Católica de Valencia (mangel.jimenez@ucv.es). Salomé MORENO NAVARRO. Profesora Asociada. Universidad Politécnica de Valencia (smoreno@upvnet.upv.es).
\end{abstract}

\section{Resumen:}

La competencia aprender a aprender (AaA) es una competencia clave (Comisión Europea [CE], 2006, 2018). Su incorporación en el sistema educativo precisa de un modelo teórico sólido y compartido por los investigadores, pero no hay acuerdo suficiente entre los académicos. Este trabajo pretende contrastar el modelo teórico elaborado por el equipo investigador con la opinión de informantes clave. Este modelo integra cinco dimensiones - cognitiva, metacognitiva, afectivo-motivacional, social-relacional y ética- y veinte subdimensiones. Las tres primeras provienen de la literatura sobre aprendizaje estratégico y autorregulado y están presentes desde los inicios de la investigación sobre el tema. La cuarta proviene del enfoque socio-cognitivo y se incorporó más tarde a los modelos explicativos. La quinta es una aportación original de este grupo. Nuestro contexto de investigación es la universidad y el objetivo contrastar nuestro modelo con informantes clave involucrados en el proceso (estudiantes, profesores, profesionales y empleadores). Para ello, se utilizó metodología cualitativa, realizándose doce grupos de discusión, uno por cada colectivo, en las tres universidades participantes en el proyecto $(\mathrm{N}=67)$. A partir de una pregunta general sobre lo que suponía la competencia AaA para los participantes, sus aportaciones fueron grabadas, transcritas y procesadas con Atlas ti.8. para cotejarlas con el modelo teórico. Se analizaron la presencia y relevancia de las dimensiones y subdimensiones estableci-

Fecha de recepción de la versión definitiva de este artículo: 04-03-2020.

Cómo citar este artículo: Gargallo López, B., García-García, F. J., López-Francés, I., Jiménez Rodríguez, M. Á. y Moreno Navarro, S. (2020). La competencia aprender a aprender: valoración de un modelo teórico | The learning to learn competence: An assessment of a theoretical model. Revista Española de Pedagogía, 78 (276), 187-211. doi: https:// doi.org/10.22550/REP78-2-2020-05

https://revistadepedagogia.org/

ISSN: 0034-9461 (Impreso), 2174-0909 (Online) 
das, prestando atención a la frecuencia de los comentarios y a sus conexiones e interrelaciones. Los resultados corroboraron el modelo propuesto por el equipo investigador: todas las dimensiones y casi todas las subdimensiones aparecieron en las intervenciones de los participantes. La siguiente fase del proceso de investigación es la construcción de un instrumento de evaluación estandarizado, a partir del modelo, para su validación estadística en estudiantes universitarios.

Descriptores: competencias clave, competencia aprender a aprender, estudiantes universitarios, aprendizaje estratégico, aprendizaje autorregulado.

\section{Abstract:}

Learning to learn (LTL) is a key competence (European Commission [EC], 2006, 2018). Building it into the educational system requires a solid theoretical model that researchers share, but there is currently a lack of agreement among academics. This work aims to check the theoretical model proposed by the research team against the opinions of key informants. This model has five dimensions - cognitive, metacognitive, affective-motivational, social-relational, andethical-aswellastwentysubdimensions. The first three dimensions come from the literature about strategic and self-regulated learning and have been present since research into this topic began. The fourth comes from the social-cognitive approach and has more recently been added into explanatory models. The fifth one is an original contribution by this group. Our research focusses on the university setting and the aim of the present work is to verify our model with some of the key informants involved in the process (students, teachers, professionals, and employers). A qualitative methodology was used, featuring twelve discussion groups, one for each group in the three participating universities $(\mathrm{N}=67)$. The participants were asked a general question about LTL and then their contributions were recorded. These contributions were then transcribed and processed using Atlas ti.8. to compare them with the theoretical model. The presence and importance of established dimensions and subdimensions was analysed, considering the frequency of comments and their connections and interrelationships. The results corroborated the model proposed by the research team: all the dimensions and almost all of the subdimensions appeared in the participants' comments. The next stage in the research process is to develop a standardised assessment instrument, based on the model, so it can be statistically validated with university students.

Keywords: key competences, learning to learn competence, university students, strategic learning, self-regulated learning.

\section{Introducción}

La competencia aprender a aprender (AaA) es una de las competencias clave para los sistemas educativos de los estados miembros de la CE (2006). En 2018 la Comisión reformuló su propuesta (CE, 2018), añadiendo a la anterior formulación de 2006 sobre lo que comporta la competencia, entre otros, algunos aspectos éticos.

A partir de la recomendación inicial, los estados han ido incorporando esas competencias 
clave en sus sistemas educativos. La meta de la UE es que el alumnado acabe la escolarización obligatoria sabiendo aprender, aunque esto es más un deseo que una realidad, de modo que aprender a aprender sigue siendo fundamental para los estudios universitarios (EI-ESU, 2012). Así se considera también desde el Espacio Europeo de Educación Superior (EEES).

\subsection{Antecedentes de la competencia}

Las propuestas de la $\mathrm{CE}$ se basan en la literatura, en el constructo aprendizaje estratégico (Danserau, 1985; Weinstein, 1987 y 1988; Weinstein y Mayer, 1985) y en el constructo aprendizaje autorregulado, cuyo nacimiento es coetáneo, fundamentándose el primero en la psicología cognitiva y en la teoría del procesamiento de la información y el segundo en el enfoque socio-cognitivo (Caena, 2019; Thoutenhoofd y Pirrie, 2013).

Típicamente se han establecido tres dimensiones que acotan los constructos aprendizaje estratégico y autorregulado: la cognitiva (manejo de habilidades, técnicas y estrategias para procesar información), la metacognitiva (conciencia/control/autorregulación de los propios procesos de aprendizaje) y la afectivo-motivacional (componentes motivacionales/actitudinales/afectivos que activan y sostienen el aprendizaje) (Hoskins y Fredriksson, 2008; Yip, 2012).

\subsection{Problema de investigación}

La evaluación de la competencia AaA es un tema clave en los estudios publicados. Es lógico, porque trabajar la competencia exige disponer de buenos instrumentos de evaluación de su logro. En nuestro país, vale la pena referir, entre otros, los trabajos de Jornet Meliá, García-Bellido y González-Such
(2012); Muñoz-San Roque, Martín-Alonso, Prieto-Navarro y Urosa-Sanz (2016); o Villardón-Gallego, Yániz, Achurra, Iraurgi y Aguilar (2013). Todos ellos focalizan su interés en la evaluación, aportando los dos últimos instrumentos estandarizados para la misma.

En Europa, existen trabajos importantes, como el de Hautamäki et al. (2002), con el objetivo de construir un marco para la evaluación, y el de Hoskins y Fredriksson (2008), que coordinaron el trabajo de la red CRELL (Center for Research on Education and Lifelong Learning) para tratar de consensuar un modelo teórico y un protocolo de evaluación. Los resultados del trabajo de esta red no satisficieron a los investigadores, pertenecientes a varios equipos de países de la UE, y no se consiguió consensuar un indicador europeo común de la competencia, quedando el tema abierto (Kupiainen, Hautamäki y Rantanen, 2008; Moreno, Cercadillo y Martínez, 2008).

De hecho, los últimos trabajos publicados muestran un desacuerdo considerable entre los académicos sobre los contenidos de esta competencia (Deakin Crick, Stringher y Ren, 2014).

No hay suficiente claridad en el aparato teórico de la competencia AaA, ni en su evaluación, y mucho menos en lo que se refiere a su trabajo en el contexto universitario. Por eso, pretendemos elaborar un modelo teórico sólido, construido desde el estudio de la literatura científica, dentro del proyecto $\mathrm{I}+\mathrm{D}+\mathrm{i}$ que estamos desarrollando ${ }^{1}$. La investigación se articula en diferentes hitos; en este texto pretendemos contrastar nuestro modelo con la valoración de los in- 
formantes clave que figuran en los Grupos de Trabajo del seguimiento del Proceso de Bolonia. Pretendemos ajustar progresivamente el modelo elaborado de cara a concretar el diseño curricular de la competencia en los grados universitarios.

\subsection{El modelo del grupo GIPU-EA}

Para llevar a cabo tal diseño curricular es imprescindible construir un buen modelo de la competencia que permita concretar su dominio (Jornet Meliá et al., 2012), integrando los componentes reconocidos en la comunidad científica.

Para ello, el Grupo de Investigación GIPU-EA (Grupo de Investigación en Pedagogía Universitaria y Estrategias de Enseñanza y Aprendizaje) revisó la literatura sobre el tema y organizó el modelo teórico en tres primeras dimensiones: cognitiva, metacognitiva y afectivo-motivacional (Hautamäki et al., 2002; Hoskins y Fredriksson, 2008; Stringher, 2014; Weinstein, Husman y Dierking, 2002; Yip, 2012). Las tres aparecen con claridad en la teoría sobre estrategias de aprendizaje (Schmeck, 1988; Weinstein, 1987; Weinstein y Mayer, 1985) y sobre aprendizaje autorregulado (Panadero, 2017; Pintrich, 2004; Zimmerman, 2000), que fundamentan la formulación de la competencia aprender a aprender.

Se incorporó una cuarta dimensión social-relacional, que proviene del enfoque socio-cognitivo (Panadero, 2017; Stringher, 2014; Thoutenhoofd y Pirrie, 2013), del aprendizaje en contexto, que recoge la relevancia del aprendizaje con los otros sujetos y de los otros, en línea de cognición situada, así como de la mejora del contexto y de la sociedad desde el propio aprendizaje. Strin- gher (2014) ubica en su modelo la dimensión social como una dimensión relevante en la línea de lo que nosotros proponemos: no solo comprensión y control de las condiciones contextuales de las tareas, sino incluyendo también valores sociales, relaciones interpersonales, aprendizaje con los otros, etc. Hautamäki et al. (2002) la sitúan también como un elemento fundamental de AaA.

Hasta aquí nuestra propuesta es coherente con los antecedentes; sin embargo, consideramos que el modelo debía ser más exigente e incluimos una quinta dimensión, ética, en relación con el aprendizaje, no contemplada anteriormente por los investigadores. Un aprendiz competente, que ha «aprendido a aprender», no puede desestimar los componentes éticos, tanto los que se refieren a la ética en el proceso de aprender, como en el proceso de usar lo aprendido para la propia mejora y la de los demás (Cortina, 2013; Grace, Innes, Patton y Stockhusen, 2017; Kass y Faden, 2018). La CE es sensible con esta perspectiva e incorpora en el texto de 2018 referencias a la dimensión ética, como dijimos antes.

Las subdimensiones de la competencia han sido propuestas a partir de las aportaciones de la literatura (Flavell y Welman, 1977; Hautamäki et al., 2002; Hautamäki y Kupiainen, 2014; Hoskins y Fredriksson, 2008; Moreno y Martín, 2014; Stringher, 2014; Pintrich, 2004; Weinstein et al., 2002; Yip, 2012; Zimmerman, 2000) y de la propia reflexión del equipo investigador.

Una descripción pormenorizada del modelo y de su construcción se puede ver en Gargallo López, Pérez-Pérez, Gar- 
cía-García, Giménez Beut y Portillo Poblador (2020). La definición de la competencia AaA que proponemos es la que sigue:

Aprender a aprender implica la capacidad de organizar y regular el propio aprendizaje de manera cada vez más eficaz y autónoma en función de los propios objetivos, del contexto y de las necesidades. Esta competencia permite la adquisición de nuevos conocimientos, habilidades y actitudes, así como resolver problemas aplicando soluciones con destreza en contextos variados, tanto a nivel personal como profesional, individualmente 0 en grupo. Conlleva ser capaz de aprender con otros y de cooperar eficazmente en la realización de tareas conjuntas. Comporta, además, conciencia de las propias habilidades y limitaciones, capacidad de planificar con eficacia las propias tareas de aprendizaje, manejando de modo eficiente los recursos, técnicas, habilidades y estrategias de aprendizaje necesarios. Supone, también, capacidad de autoevaluar y autorregular el propio desempeño a nivel cognitivo y afectivo, optimizando las capacidades para superar los obstáculos y limitaciones. Implica, así mismo, tener curiosidad intelectual, motivación intrínseca e interés para afrontar las dificultades y superarlas con éxito, manteniendo un compromiso ético y una actitud positiva hacia el aprendizaje.

En el gráfico y en las tablas que siguen incluimos la descripción de la competencia: el Gráfico 1 presenta las dimensiones (D) propuestas, con su descripción. En las Tablas 1-5, se recogen las 20 subdimensiones (S) componentes y su descripción (Des) (50 descripciones).

Gráfico 1. Dimensiones de la competencia AaA.

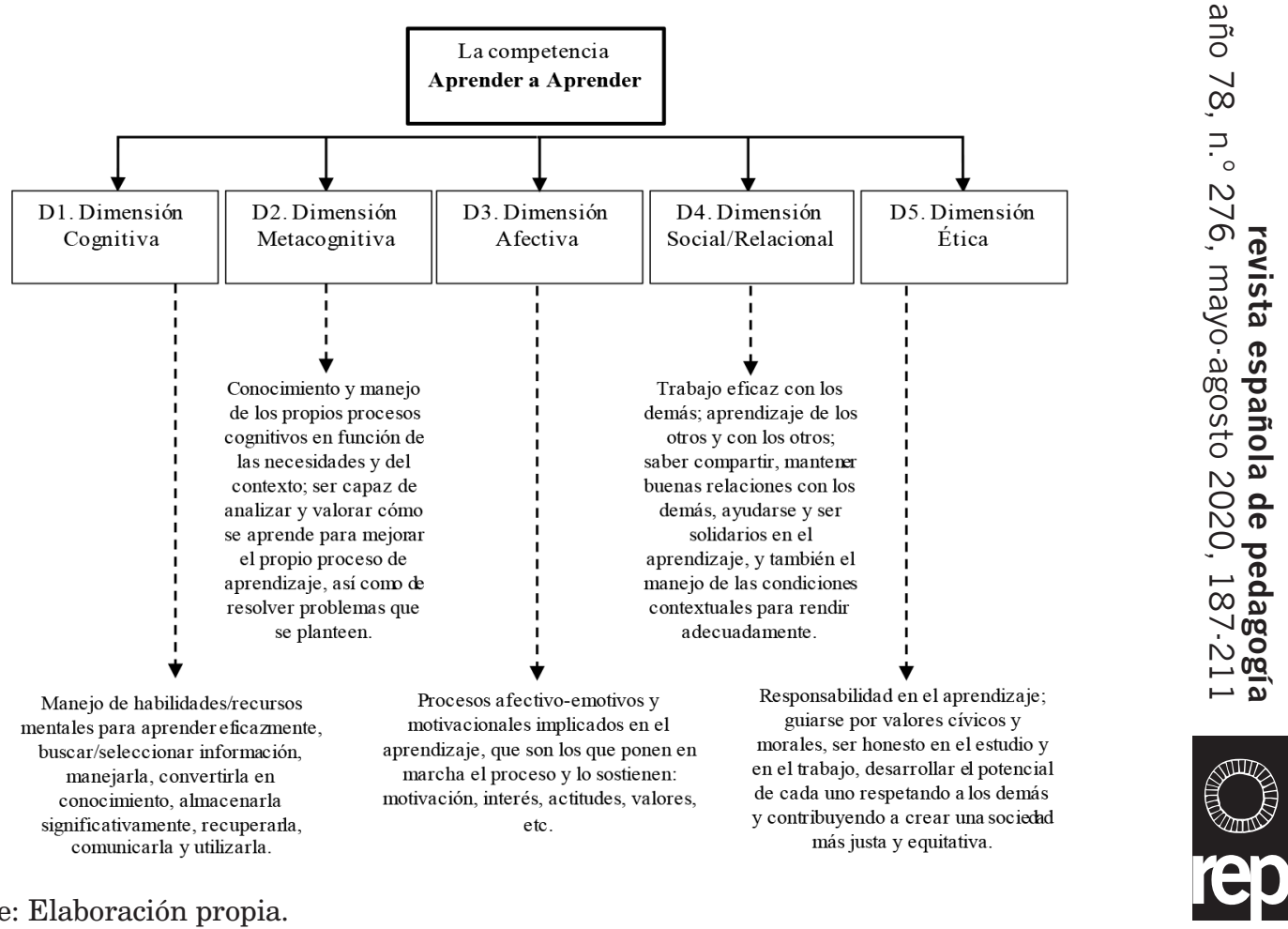

Fuente: Elaboración propia. 
TABLA 1. Subdimensiones de la dimensión cognitiva.

\begin{tabular}{|c|c|c|}
\hline \multicolumn{3}{|c|}{ DIMENSIÓN COGNITIVA } \\
\hline Subdimensiones & & Descripciones/Componentes \\
\hline \multirow{2}{*}{$\begin{array}{l}\text { S1. Gestión } \\
\text { eficaz de la } \\
\text { información }\end{array}$} & & $\begin{array}{l}\text { Des1. Usar fuentes de información relevantes. Buscar y selec- } \\
\text { cionar información, gestionarla adecuadamente, transformarla } \\
\text { en conocimiento, almacenarla comprensivamente y recuperarla } \\
\text { eficazmente para su utilización. }\end{array}$ \\
\hline & & $\begin{array}{l}\text { Des2. Utilizar y transferir lo aprendido a otros contextos (vida } \\
\text { cotidiana, resolución de problemas del ámbito de estudios y } \\
\text { profesional, etc.). }\end{array}$ \\
\hline \multirow{9}{*}{$\begin{array}{l}\text { S2. Habilidades } \\
\text { de comunica- } \\
\text { ción }\end{array}$} & \multirow[t]{4}{*}{$\begin{array}{l}\text { S2.1. Habilidades de } \\
\text { comunicación oral }\end{array}$} & $\begin{array}{l}\text { Des3. Comunicarse eficazmente a nivel oral, trasmitir conoci- } \\
\text { mientos y expresar ideas de manera clara, rigurosa y convincen- } \\
\text { te. }\end{array}$ \\
\hline & & $\begin{array}{l}\text { Des4. Expresarse oralmente de forma estructurada e inteligible } \\
\text { tanto en presentaciones orales breves como en intervenciones } \\
\text { largas con debate posterior. }\end{array}$ \\
\hline & & Des5. Argumentar y contraargumentar adecuadamente. \\
\hline & & Des6. Adaptarse al contexto y a la audiencia. \\
\hline & \multirow[t]{3}{*}{$\begin{array}{l}\text { S2.2. Habilidades de } \\
\text { comunicación escrita }\end{array}$} & $\begin{array}{l}\text { Des7. Comunicarse eficazmente a nivel escrito, trasmitir conoci- } \\
\text { mientos y expresar ideas de manera clara, rigurosa y convincen- } \\
\text { te. }\end{array}$ \\
\hline & & $\begin{array}{l}\text { Des8. Expresarse por escrito con orden lógico y con buena cons- } \\
\text { trucción, elaborando documentos bien estructurados ajustados a } \\
\text { diferentes finalidades. }\end{array}$ \\
\hline & & $\begin{array}{l}\text { Des9. Escribir textos rigurosos, de calidad científica y técnica, } \\
\text { relacionados con el campo de estudio, informes, TFG, etc. }\end{array}$ \\
\hline & $\begin{array}{l}\text { S2.3. Conocimiento y } \\
\text { manejo de lenguajes } \\
\text { no verbales }\end{array}$ & $\begin{array}{l}\text { Des10. Conocer y manejar eficazmente lenguajes diferentes del } \\
\text { verbal: visual, icónico, artístico, etc., en cuanto a que se usan } \\
\text { como vehículo de aprendizaje. }\end{array}$ \\
\hline & $\begin{array}{l}\text { S2.4. Conocimiento } \\
\text { y manejo de idiomas } \\
\text { extranjeros }\end{array}$ & $\begin{array}{l}\text { Des11. Manejar idiomas extranjeros para comunicarse con flui- } \\
\text { dez y para aprender. }\end{array}$ \\
\hline $\begin{array}{l}\text { S3. Manejo } \\
\text { de TICS }\end{array}$ & & $\begin{array}{l}\text { Des12. Aplicar eficazmente las TIC en el aprendizaje y en la } \\
\text { actividad profesional (procesadores de textos, hoja de cálculo, } \\
\text { software de presentaciones, paquetes estadísticos en los grados } \\
\text { en que sea preciso, buscadores y bases de datos, relacionadas con } \\
\text { los estudios y la profesión, etc.). }\end{array}$ \\
\hline \multirow{4}{*}{$\begin{array}{l}\text { S4. Pensamiento } \\
\text { crítico y } \\
\text { creativo }\end{array}$} & & $\begin{array}{l}\text { Des13. Elaborar propuestas personales creativas yendo más allá } \\
\text { de lo dado. }\end{array}$ \\
\hline & & $\begin{array}{l}\text { Des14. Realizar análisis, inferencias y razonamientos críticos } \\
\text { diferenciados sobre tareas y situaciones de la vida. }\end{array}$ \\
\hline & & $\begin{array}{l}\text { Des15. Cuestionar los supuestos subyacentes en nuestras formas } \\
\text { habituales de pensar y actuar. }\end{array}$ \\
\hline & & $\begin{array}{l}\text { Des16. Producir pensamiento nuevo - pensar diferente- sobre } \\
\text { la realidad en que uno se desempeña. }\end{array}$ \\
\hline
\end{tabular}

Fuente: Elaboración propia. 
TABLA 2. Subdimensiones de la dimensión cognitiva.

\begin{tabular}{|c|c|}
\hline \multicolumn{2}{|r|}{ DIMENSIÓN METACOGNITIVA } \\
\hline Subdimensiones & Descripciones/Componentes \\
\hline \multirow{4}{*}{$\begin{array}{l}\text { S5. Conocimiento } \\
\text { de sí mismo, } \\
\text { de la tarea y de } \\
\text { las estrategias } \\
\text { para abordarla }\end{array}$} & Des17. Identificar las propias destrezas y limitaciones para mejorarlas en lo posible. \\
\hline & $\begin{array}{l}\text { Des18. Pensar sobre la tarea y analizarla, sobre los objetivos y las estrategias necesarias para su abordaje } \\
\text { y solución. }\end{array}$ \\
\hline & Des19. Cuestionarse los objetivos de aprendizaje y formularse objetivos propios. \\
\hline & $\begin{array}{l}\text { Des20. Tomar decisiones sobre qué y cómo aprender, en función de los objetivos y necesidades propias y/o del } \\
\text { desempeño profesional. }\end{array}$ \\
\hline \multirow{2}{*}{$\begin{array}{l}\text { S6. Planificación, } \\
\text { organización } \\
\text { y gestión del } \\
\text { tiempo }\end{array}$} & $\begin{array}{l}\text { Des21. Realizar una planificación adecuada de las tareas para alcanzar los objetivos previstos a corto, medio y } \\
\text { largo plazo, en función del contexto y el tiempo disponible. }\end{array}$ \\
\hline & Des22. Priorizar, jerarquizar, organizar las actividades necesarias y realizarlas. \\
\hline \multirow{2}{*}{$\begin{array}{l}\text { S7. Autoevalua- } \\
\text { ción, control, } \\
\text { autorregula- } \\
\text { ción }\end{array}$} & $\begin{array}{l}\text { Des23. Analizar, evaluar y monitorizar el propio desempeño estableciendo los mecanismos necesarios para } \\
\text { mejorar la ejecución e introduciendo los ajustes necesarios, tanto en la planificación como en la implementa- } \\
\text { ción, mediante el uso de estrategias y habilidades más eficientes. }\end{array}$ \\
\hline & Des24. Buscar orientación, asesoramiento y apoyo si se precisa. \\
\hline $\begin{array}{l}\text { S8. Resolución de } \\
\text { problemas }\end{array}$ & $\begin{array}{l}\text { Des25. Analizar y resolver problemas de forma efectiva y contextualizada, identificando y definiendo los } \\
\text { elementos significativos que los constituyen, desarrollando procesos de razonamiento complejo de alto nivel, } \\
\text { no simplemente acciones asociativas y rutinarias. }\end{array}$ \\
\hline
\end{tabular}

Fuente: Elaboración propia.

\section{TABLA 3. Subdimensiones de la dimensión afectiva/motivacional.}

\begin{tabular}{|c|c|}
\hline \multicolumn{2}{|r|}{ LA DIMENSIÓN AFECTIVA Y MOTIVACIONAL } \\
\hline Subdimensiones & Descripciones/Componentes \\
\hline \multirow{4}{*}{$\begin{array}{l}\text { S9. Motivación } \\
\text { y actitud } \\
\text { positiva ante el } \\
\text { aprendizaje y } \\
\text { la mejora }\end{array}$} & $\begin{array}{l}\text { Des26. Desarrollar y mantener la motivación, la curiosidad, el interés y el gusto por comprender los conteni- } \\
\text { dos y aprender a fondo. }\end{array}$ \\
\hline & $\begin{array}{l}\text { Des27. Desarrollar una voluntad firme para aprender, orientando la acción en la dirección oportuna para } \\
\text { lograr buenos resultados. }\end{array}$ \\
\hline & Des28. Perseverar en el aprendizaje, concentrarse durante períodos prolongados de tiempo, superar las dificultades. \\
\hline & Des29. Tolerar la frustración cuando no se consigue el éxito en el aprendizaje. Resiliencia. \\
\hline $\begin{array}{l}\text { S10. Atribuciones } \\
\text { internas }\end{array}$ & Des30. Atribuir los resultados del aprendizaje y el rendimiento al propio esfuerzo que uno controla y maneja. \\
\hline \multirow{2}{*}{$\begin{array}{l}\text { S11. Autoconcepto } \\
\text { y autoestima } \\
\text { Autoeficacia }\end{array}$} & $\begin{array}{l}\text { Des31. Tener una imagen ajustada de sí mismo, aceptarse y apreciarse, lo que es compatible con la conciencia } \\
\text { de las propias limitaciones y con el esfuerzo por mejorar. }\end{array}$ \\
\hline & $\begin{array}{l}\text { Des32.Incrementar la autoeficacia, sentirse capaz de conseguir objetivos exigentes y de tener éxito al realizar } \\
\text { las tareas. }\end{array}$ \\
\hline \multirow{2}{*}{$\begin{array}{l}\text { S12. Bienestar } \\
\text { físico y } \\
\text { emocional }\end{array}$} & Des33. Mantener un buen tono físico y emocional, apropiado para aprender y trabajar. \\
\hline & Des34. Llevar una vida saludable (alimentación, descanso, sueño y ejercicio) que ayude al aprendizaje. \\
\hline \multirow{2}{*}{$\begin{array}{l}\text { S13. Autorregula- } \\
\text { ción emocio- } \\
\text { nal y control } \\
\text { de la ansiedad }\end{array}$} & $\begin{array}{l}\text { Des35. Observar, analizar y modificar las reacciones emocionales de una forma socialmente aceptable en } \\
\text { función de los objetivos de aprendizaje. }\end{array}$ \\
\hline & $\begin{array}{l}\text { Des36. Controlar la ansiedad, aprender a relajarse en situaciones de estrés (exámenes, exposiciones, inter- } \\
\text { venciones públicas, etc.). }\end{array}$ \\
\hline
\end{tabular}

Fuente: Elaboración propia. 
TABLA 4. Subdimensiones de la dimensión social-relacional.

\begin{tabular}{|c|c|}
\hline \multicolumn{2}{|r|}{ DIMENSIÓN SOCIAL/RELACIONAL } \\
\hline Subdimensiones & Descripciones/Componentes \\
\hline \multirow[t]{2}{*}{ S14. Valores sociales } & Des37. Valorar la relación interpersonal y el trabajo con los otros para aprender con y de ellos. \\
\hline & $\begin{array}{l}\text { Des38. Trabajar, estudiar y esforzarse para aportar al conjunto de la sociedad, no solo para el propio desa- } \\
\text { rrollo personal. }\end{array}$ \\
\hline \multirow{3}{*}{$\begin{array}{l}\text { S15. Actitudes de } \\
\text { cooperación } \\
\text { y solidaridad; } \\
\text { relaciones in- } \\
\text { terpersonales }\end{array}$} & $\begin{array}{l}\text { Des39. Cooperar eficazmente con los compañeros para: resolver problemas, estudiar, aprender con y de ellos } \\
\text { y trabajar. }\end{array}$ \\
\hline & Des40. Establecer y mantener buenas relaciones interpersonales con compañeros y profesores. \\
\hline & $\begin{array}{l}\text { Des41. Negociar, compartir, argumentar respetando las opiniones ajenas, etc., desarrollando las habilidades } \\
\text { sociales necesarias: escucha, empatía, asertividad y solidaridad en la relación con los otros. }\end{array}$ \\
\hline \multirow[t]{2}{*}{$\begin{array}{l}\text { S16. Trabajo en } \\
\text { equipo }\end{array}$} & $\begin{array}{l}\text { Des42. Realizar tareas compartiendo objetivos e intereses, superando las dificultades que se presenten } \\
\text { para ello. }\end{array}$ \\
\hline & $\begin{array}{l}\text { Des43. Participar activamente en grupos de trabajo aportando ideas y esfuerzos, recibiendo ayuda y dándo- } \\
\text { la, liderando cuando sea preciso, etc. }\end{array}$ \\
\hline \multirow{2}{*}{$\begin{array}{l}\text { S17. Control } \\
\text { condiciones } \\
\text { ambientales }\end{array}$} & $\begin{array}{l}\text { Des44. Crearse un ambiente adecuado para trabajar y rendir que permita la concentración y que disponga } \\
\text { de medios y recursos necesarios. }\end{array}$ \\
\hline & Des45. Modular los elementos del contexto para aprender mejor. \\
\hline
\end{tabular}

Fuente: Elaboración propia.

TABLA 5. Subdimensiones de la dimensión ética.

\begin{tabular}{|c|c|}
\hline \multicolumn{2}{|r|}{ DIMENSIÓN ÉTICA } \\
\hline Subdimensiones & Descripciones/Componentes \\
\hline \multirow{2}{*}{$\begin{array}{l}\text { S18. Responsabi- } \\
\text { lidad en el } \\
\text { aprendizaje }\end{array}$} & $\begin{array}{l}\text { Des46. Mantener actitud responsable hacia el aprendizaje aprovechando el tiempo y los recursos dispo- } \\
\text { nibles. }\end{array}$ \\
\hline & $\begin{array}{l}\text { Des47. Esforzarse por un trabajo eficaz y comprometido, por hacer las cosas lo mejor posible, evitando el } \\
\text { trabajo incompleto y/o mal hecho. }\end{array}$ \\
\hline \multirow{2}{*}{$\begin{array}{l}\text { S19. Actitudes y } \\
\text { valores cívicos } \\
\text { y morales }\end{array}$} & Des48. Ser honesto, responsable, respetuoso con los otros y veraz. Evitar las malas prácticas (plagio, etc.). \\
\hline & $\begin{array}{l}\text { Des } 49 \text {. Trabajar para el bien propio y para el bien común, contribuyendo a avanzar hacia una sociedad } \\
\text { más justa y equitativa. }\end{array}$ \\
\hline $\begin{array}{l}\text { S20. Respeto a los } \\
\text { códigos éticos y } \\
\text { deontológicos }\end{array}$ & $\begin{array}{l}\text { Des50. Integrar en el ejercicio profesional los códigos éticos y deontológicos: respetar los derechos huma- } \\
\text { nos, trabajar con rigor, respetar el secreto profesional, no abusar de la propia posición cuando se tiene } \\
\text { poder, etc. }\end{array}$ \\
\hline
\end{tabular}

Fuente: Elaboración propia.

\section{Método}

\subsection{Objetivos}

El objetivo de este trabajo era contrastar el modelo de la competencia elaborado por el equipo investigador con la perspectiva de cuatro grupos de informantes clave involucrados en el proceso. Se buscaba refrendar el modelo con las aportaciones de cuatro colectivos considerados como interesados (stakeholders) en el proceso de Bolonia (empleadores, profesionales, profesorado y alumnado).

Era sumamente importante recibir feedback de cuatro de los principales grupos de personas interesadas en la 
formación impartida en la universidad, que vienen recogidos con claridad en documentos relevantes del proceso de convergencia de Bolonia, en concreto en el Tuning (González y Wagenaar, 2006) $y$ en el marco europeo de cualificaciones (Grupo de trabajo de Bolonia sobre marcos de cualificaciones, 2005). Estos cuatro grupos son profesores y alumnos, como agentes implicados en el proceso de formación, y empleadores y profesionales, como agentes relevantes del mundo laboral; los unos por ser los responsables de la selección y contratación de los egresados y tener conciencia de la formación que necesitan para incorporarse al mundo laboral, y los otros por tener experiencia del mundo del trabajo y de sus necesidades.

\subsection{Diseño}

El proyecto de investigación en que se inscribe este trabajo tiene varios objetivos, por lo que usa un diseño de métodos mixtos, integrando métodos cuantitativos y cualitativos (Brannen, 2017; Creswell, 2010). Sin embargo, el presente trabajo se limita a una parte del diseño, cualitativa, utilizando grupos focales para recoger información relevante (Merriam y Grenier, 2019).

Nos centramos en el estudio de las percepciones de informantes clave involucrados en el proceso: empleadores, profesorado universitario, alumnado de últimos cursos y profesionales, para contrastar el modelo teórico elaborado.

Se han realizado tres grupos de discusión para cada uno de los colectivos enumerados, en las tres ramas de conocimiento en que se ha realizado la investi gación: Ciencias de la Educación (Universidad Católica de Valencia), Ciencias de la Salud (Universidad de Valencia) e Ingeniería (Universidad Politécnica de Valencia); doce en total.

\subsection{Muestra}

La muestra se ha estructurado con un muestreo intencional, a partir de los siguientes criterios:

- Pertenencia a las tres ramas de conocimiento involucradas.

- Tipología y calidad de los informantes clave:

- Empleadores y/0 responsables de departamentos de recursos humanos de las empresas (relacionadas con la educación, con las ciencias de la salud y con las ingenierías).

- Profesionales interesados (de las tres ramas).

- Profesorado universitario de las tres ramas de conocimiento sensibilizado con el tema y con más de 20 años de experiencia.

- Alumnado de los últimos cursos con buen rendimiento académico (con media de 8.50 superior).

- Disposición a participar en el estudio.

La muestra prevista, 4-6 sujetos por grupo, se concretó como aparece en la tabla: 
Bernardo GARGALLO, Fran J. GARCÍA, Inmaculada LÓPEZ, Miguel A. JIMÉNEZ y Salomé MORENO

TABla 6. Participantes.

\begin{tabular}{|l|c|c|c|c|c|c|c|c|c|c|}
\hline & \multicolumn{3}{|c|}{$\begin{array}{c}\text { UVEG } \\
\text { (CC. Salud) }\end{array}$} & \multicolumn{3}{c|}{ UPV (Ingenierías) } & \multicolumn{3}{c|}{$\begin{array}{c}\text { UCV } \\
\text { (CC. Educación) }\end{array}$} & Total \\
\hline & Total & Varones & Mujeres & Total & Varones & Mujeres & Total & Varones & Mujeres & \\
\hline Empleadores* & 2 & 0 & 2 & 8 & 2 & 6 & 4 & 0 & 4 & 14 \\
\hline Profesionales & 6 & 2 & 4 & 7 & 4 & 3 & 5 & 1 & 4 & 18 \\
\hline Profesorado & 6 & 3 & 3 & 8 & 4 & 4 & 5 & 0 & 5 & 19 \\
\hline Alumnado & 6 & 4 & 2 & 6 & 4 & 2 & 4 & 1 & 3 & 16 \\
\hline Total & 20 & & & 29 & & & 18 & & & 67 \\
\hline
\end{tabular}

* Habían sido citados cuatro empleadores, pero solo pudieron asistir a la reunión de trabajo dos, ambas mujeres.

Fuente: Elaboración propia.

\subsection{Instrumentos}

Se elaboró un protocolo para los grupos de discusión, con una sola pregunta, precedida de la afirmación siguiente:

Cualquier persona y cualquier profesional necesita seguir aprendiendo para mejorar, adaptarse y realizar sus funciones de modo eficaz en un contexto socioeconómico y cultural cambiante. En este contexto, la Unión Europea plantea como una de las competencias básicas que deben adquirir los estudiantes en el sistema educativo la de aprender a aprender.

Desde su punto de vista y en relación con el aprender a aprender, con esta necesidad de seguir aprendiendo, iqué cualidades, capacidades, habilidades, etc., debe adquirir o perfeccionar una persona durante sus estudios de grado en la universidad para convertirse en un profesional competente y responsable?

Se prefirió este planteamiento abierto frente a otras posibilidades, como presentar a los participantes el modelo del equipo investigador para su valoración. La información recogida fue rica y susceptible de tratamiento.

\subsection{Procedimiento}

A partir del estudio de la literatura, el equipo investigador elaboró un modelo teórico de la competencia (apartado 1.3), que, sometido al juicio de expertos y revisado, quedó como se presenta en las tablas.

Posteriormente, se llevaron a cabo los grupos de discusión en las tres universidades, grabando las intervenciones. Las transcripciones fueron incorporadas como documentos primarios al software Atlas. ti.8 creando una unidad hermenéutica y evitando automatizar el proceso de análisis (Wolf y Silver, 2017).

Para el análisis de resultados se trabajó con los materiales recogidos, procediendo al establecimiento de categorías. El trabajo se realizó mediante cuatro jueces entrenados, miembros del equipo investigador, prestando especial atención al proceso de triangulación de fuentes (Denzin y Lincoln, 2004; Wolf y Silver, 2017).

El procesamiento de datos constó de dos etapas interdependientes. Primero 
se trabajó a nivel textual con los datos brutos, señalando segmentos para codificar en función de una lista de códigos sobre las categorías de análisis prefijadas. Después, se vincularon los códigos a nivel conceptual con base en los elementos discursivos y en el modelo teórico, creando así un mapa de relaciones entre elementos para corroborar, refutar y complementar el modelo teórico inicial. Todo ello permitió crear redes conceptuales, tomando las dimensiones previstas en el modelo como categorías centrales o familias: cognitiva (D1), metacognitiva (D2), afectiva-motivacional (D3), social-relacional (D4) y ética (D5). Para establecer los nodos secundarios se usaron las subdimensiones del modelo, generando Grupos con códigos rotulados «S1», «S2», etc., y su respectiva denominación (Gráfico 2) ${ }^{2}$ y para establecer los nodos terciarios se utilizaron las descripciones/componentes de dichas subdimensiones con códigos rotulados «Des1», «Des2», etc.

\section{Resultados}

Se presentan los resultados estudiando primero las relaciones dentro de cada dimensión, para analizar su coherencia intradimensional, y, luego, las relaciones entre las dimensiones establecidas por los participantes, para analizar la coherencia interdimensional del modelo.

En el Gráfico 2 se presentan los resultados de los 12 grupos con las cinco dimensiones y subdimensiones del modelo y con las puntuaciones de enraizamiento (e), que indican el número de veces que ha aparecido en el texto un comentario de los participantes coherente con la descripción correspondiente y las de densidad (d), que se refieren a las veces que los participantes establecen relaciones entre la descripción correspondiente y otras mencionadas en el grupo, en cada caso. Ambos datos son relevantes. Las dimensiones y subdimensiones son elaboraciones teóricas del modelo que se somete a prueba, mientras que los comentarios relacionados con las descripciones/componentes de las mismas - recogidas en el gráfico bajo la denominación de «Des»son aportaciones de los participantes en los grupos y se vinculan a variables del modelo teórico.

En la presentación de los resultados se intercalan testimonios de los participantes, citados en función del documento primario al que se refieren: DP1, DP2 y DP3 (estudiantes), DP4, DP5 y DP6 (empleadores), DP7, DP8 y DP9 (profesionales) y DP10, DP11 y DP12 (profesorado). En estos testimonios literales se indica el identificador que proporciona el Atlas.ti.8 tras el documento primario, precedido por dos puntos (:).

\subsection{Coherencia intradimensional}

La dimensión cognitiva (D1) fue la más mencionada (e=98). La subdimensión S1 («gestión eficaz de la información») lo fue en 31 ocasiones. Le siguió la S2 («habilidades de comunicación», e=35) (S2.1, «habilidades de comunicación oral», e=24; $\mathrm{S} 2.2$, «habilidades de expresión escrita», $e=8$; S2.3, "conocimiento de idiomas extranjeros», e=3; la S2.3 no fue mencionada («conocimiento y manejo de lenguajes no verbales»); la S3 (TICs) apareció 4 veces y 
la S4 («pensamiento crítico y creativo») 28. Además, la D1 obtuvo la mayor cantidad de vínculos internos $(\mathrm{d}=12)$. Esto puede tener que ver con que sea una de las más consolidadas y que aparecen primero en las investigaciones sobre el aprendizaje. Calculando su nivel de relaciones internas a partir del análisis cualitativo, se relativizó el número de asociaciones entre las subdimensiones cognitivas que establecieron los participantes, obteniéndose un valor de $.583^{3}$. La «comunicación eficaz a nivel oral» (Des3) y las «propuestas personales creativas» (Des13) fueron las descripciones/componentes que más contribuyeron a garantizar la conexión interna de esta dimensión, aunque sus densidades intradimensionales, que marcan la relación que los intervinientes establecen entre los componentes, no fueron demasiado elevadas ( $d=2$, en ambos casos). La segunda de ellas también arrojó valores de enraizamiento notables $(e=10)$, comparados con los otros componentes cognitivos. Únicamente fueron superados por la «búsqueda, selección y gestión eficaz de la información» $(e=19)$.

La dimensión metacognitiva (D2) fue la segunda con mayor valor de enraizamiento $(e=76)$. «Cuestionarse objetivos de aprendizaje y plantearse objetivos propios» (Des19) fue la idea que más conectó sus componentes, con 3 asociaciones dentro de la dimensión: con «conocer destrezas y limitaciones» (Des17), con «analizar, evaluar...» (Des23), y con «resolución de problemas...» (Des25). Por otra parte, esta descripción/componente (Des19), junto con «conocer destrezas y limitaciones...» (Des17) (componentes de la subdimensión S6), fueron las más mencio- nadas en los grupos ( $\mathrm{e}=11 \mathrm{y}$ e $=10$ respectivamente). Hubo algunos testimonios interesantes de los profesores que muestran estas conexiones:

un alumno tiene que dejar de ser un elemento pasivo para convertirse en un elemento activo y ser constructor de su propio aprendizaje. Para que uno construya $[. .$.$] tiene que [...] acercar [...] el conte-$ nido teórico que está viendo a la realidad. Resolviendo problemas reales acercamos [...] al alumno a resolver un problema que [...] se le presenta hoy, y hoy puede equivocarse porque está en la universidad, pero, a lo mejor, el día de mañana [...] no lo va a poder hacer (DP11:3).

La subdimensión más mencionada fue la $\mathrm{S} 6$, de conocimiento de sí mismo... $(\mathrm{e}=35)$, pero hay que reseñar que de todas las descripciones/componentes de la dimensión D2, fue la Des25, «resolución de problemas», la que tuvo un enraizamiento mayor ( $\mathrm{e}=26$ ), superando a cualquier otra descripción de esta subdimensión.

La dimensión afectivo-motivacional (D3) apareció 64 veces en los comentarios. La idea más mencionada fue la «motivación y curiosidad por aprender» (descripción/componente Des26) (e=26). Esta idea, aglutinada en la subdimensión S10, también contribuyó más que cualquier otra a la conexión interna $(\mathrm{d}=3)$, relacionándose con las «atribuciones internas» (Des30), con la «imagen y conciencia ajustada de uno mismo» (Des31) y con la «autoeficacia» (Des32). Otras descripciones/ componentes fueron mencionados, también, en un segundo nivel de importancia, «tolerar la frustración...» (Des29) y «tener 
una imagen y conciencia de uno mismo..." (Des 31 ) (e=14 y e=11 respectivamente). En palabras de los empleadores, los estudiantes «no son capaces de darle valor a todo lo que saben y a todo el potencial que tienen» (DP6:10-11).

La dimensión social/relacional (D4) fue mencionada 66 veces en los testimonios de los participantes. Esta dimensión obtuvo un valor relativo de .833 , siendo la de mayor conexión interna relativa. No es de las dimensiones más clásicas en la literatura, siendo muchas de las descripciones/componentes que la integran debidas a los avances en el aprendizaje social de los años 90 . Nuestro equipo amplió a propósito el contenido de esta dimensión a partir de las aportaciones de la teoría del aprendizaje social de Bandura, incorporando también el resultado de posteriores estudios que ahondaban en la cooperación, el trabajo en grupos y otros aspectos similares. Quizás la conexión interna de las dimensiones guarde relación con su consolidación en el historial de estudios publicados desde el siglo pasado.

La subdimensión con mayor enraizamiento fue la S16, de actitudes de cooperación y solidaridad $(\mathrm{e}=27)$-dentro de esta fue la descripción/componente «habilidades sociales», la más referida, e=18-. Le siguió la S17, «trabajo en equipo» $(\mathrm{e}=23)$ y, por fin, la S15, «valores sociales» $(\mathrm{e}=16)$.

En esta dimensión, la descripción/componente Des42, «trabajar en equipo» tuvo el mayor poder de conexión $(\mathrm{d}=4)$, relacionándose con «valorar las relaciones interpersonales» (Des37), con «cooperar con compañeros» (Des39), con «habilidades sociales» (Des41) y con «trabajar aportando ideas...» (Des43). Otras dos descripciones/componentes tuvieron dos conexiones cada una: «cooperar con los compañeros» (Des39), relacionada con Des42 y Des43; y «trabajar aportando ideas, dando y pidiendo ayuda...» (Des43), relacionada con Des39 y Des42 . Los estudiantes dijeron que:

Algo importantísimo también será entrenar para saber trabajar en equipo porque somos muy individualistas y [...] por querer [...] llegar al objetivo, te saltas fases y no esperas a que los demás lleguen, trabajando de forma más sosegada, más tranquila. Cada alumno va a tener su tiempo, su proceso (DP1:38).

«Trabajar en equipo» (Des42) obtuvo un enraizamiento de 17, pero las «habilidades sociales» (Des41) fueron mencionadas en 18 ocasiones. Esto es interesante porque las «habilidades sociales» no contribuyeron tanto a la conexión interna como otras subdimensiones, ya que este componente solo tuvo una densidad de 3 dentro de esta dimensión. «Valorar las relaciones interpersonales...» también tuvo presencia relevante entre los participantes $(\mathrm{e}=13)$.

La dimensión ética (D5) fue mencionada en menos ocasiones. Así y todo, aparecieron 52 referencias $(e=52)$, lo que da idea de su importancia para los participantes.

La subdimensión con mayor enraizamiento fue la S20, «actitudes y valores cívicos y morales» $(e=26)$, seguida por la S19, «responsabilidad en el aprendizaje» (e=18), y por la S21, "códigos éticos y deontológicos» $(e=8)$. Las descripciones que más comentarios merecieron fueron 
«ser honesto, responsable y respetuoso» (Des48) y «trabajar por el bien propio y común» (Des49), ambas componentes de la S20 $(e=13)$.

Esta dimensión es la que menos conexiones presenta a nivel interno. La descripción Des50, «códigos éticos y deontológicos» $(\mathrm{d}=2)$ se relacionó con la Des48 («ser honesto, responsable...») y con la Des49 («trabajar por el bien propio y el bien común»). Los empleadores dijeron que:

La tolerancia en el profesional [...] es súper importante. [...] No hablamos de justicia, sino de equidad. [...] otro concepto que yo veo que tampoco [...] han trabajado [...] es el tema de la integridad [...] de la persona y [...] del líder, fundamentalmente (DP4:19-20-21).

\subsection{Coherencia entre dimensiones}

Las relaciones externas entre dimensiones corroboran la coherencia del modelo, presentando un entramado consistente.

La dimensión cognitiva (D1) se relacionó con la metacognitiva (D2): «buscar, seleccionar y gestionar información eficazmente» interaccionó con la «reflexión sobre qué, cómo y para qué aprender» y con «analizar, evaluar y monitorizar para mejorar la ejecución». El profesorado comentó:

hay que enseñarles a que filtren, [...] que conozcan fuentes [...] fiables [...] que se conozcan y luego tener [...] visión crítica [...]. Que tenga el método y el orden, y los pasos que tienen que seguir, que sea capaz de ser autónomo y de ir solucionando ese problema (DP12:8).

La cognitiva se relacionó también con la afectiva: se asoció «comunicarse efi- cazmente a nivel oral» con «atribuciones internas», considerando que «hay que promover [...] que cada uno pueda seguir trabajando eso y que tengas confianza» (DP6:33).

La dimensión metacognitiva (D2) fue la dimensión más conectada a nivel externo. En los comentarios de los participantes la «resolución de problemas» (componente metacognitivo) se asoció con el «análisis, inferencia..." (Des14) y con "cuestionar supuestos subyacentes» (Des15) (descripciones/componentes cognitivos). Los profesionales afirmaban que:

se centran solo en la aplicación de técnicas [...] les faltan [...] habilidades a la hora de realizar autocrítica 0 cómo buscar una solución a un determinado problema. Es como que continuamente necesitan un supervisor $[\ldots]$ no tienen esa independencia a la hora de trabajar (DP8: 6-7).

«Conocer destrezas y limitaciones propias» (componente cognitivo) se asoció con «tolerar la frustración...» y con una «imagen y conciencia de uno mismo ajustadas» (componentes afectivos). Los empleadores dijeron que «es importante cómo digieren el fracaso [...] porque ante él [...] se bloquean» (DP7:22). Los alumnos afirmaron que:

una capacidad importante es conocerse a sí mismo, porque cada uno tiene [...] una forma de estudiar [...] conocerse a uno mismo te ayuda [...] a optimizar el tiempo [...] y luego, en el futuro también te servirá para adquirir nuevos conocimientos (DP1:21).

También la dimensión social/relacional (D4) se asoció con la metacognitiva (D2): «valorar relaciones interpersonales 
y el trabajo con otras personas...» (componente social) se relacionó con «priorizar, jerarquizar y organizar actividades» y con «analizar, evaluar y monitorizar...» (ambos componentes metacognitivos). En palabras de los empleadores:

tienen que saber aprender a gestionar el tiempo [...]ser personas empáticas [...] cada persona es un mundo diferente y tenemos que conocer ese mundo para [...] sacar de ellos las estrategias para que sigan aprendiendo y puedan afrontar diferentes situaciones en diferentes contextos [...] para orientarla y que se atreva a poner en marcha las estrategias que necesita, pero desde donde está y desde cómo es (DP6:4).

La dimensión afectiva (D3) se relacionó con la social/relacional (D4): la «motivación y curiosidad por aprender» (afectiva) con el «trabajo en equipo» (social) y las «atribuciones internas» (afectiva) con «trabajar aportando ideas, dando y pidiendo ayuda...» (social):

si tú trabajas en grupo para querer aprender más sobre un tema [...] si tú no te conoces a ti mismo y lo que puedes ofrecer a ese grupo, y lo que ese grupo te puede ofrecer a ti [...] no puedes abarcar todas las posibilidades que te ofrece, porque [...] si no te conoces para seguir avanzando, estás limitado (DP2:15).

Por fin, la dimensión social/relacional (D4) se relacionó con la ética (D5): «cooperar con los compañeros» (social) con «esforzarse por un trabajo bien hecho» (ética).

Se dijo que hay que «hacerse responsable de sus errores, ya que hay una [...] tendencia de echarle la culpa a otros [...] eso es una de las grandes situaciones de fracaso en los colectivos de trabajo» (DP6:26).

También se relacionaron las «habilidades sociales» (social) con ser «honesto, responsable y respetuoso» y con los «códigos éticos y deontológicos...» (ética).

La Tabla 7 recoge las relaciones externas que se hallaron en los grupos y permite identificar las subdimensiones ligadas a una determinada dimensión y las subdimensiones con más relaciones externas. La dimensión metacognitiva es la que más relaciones externas presenta (8), seguida de la social (7), cognitiva (4), afectiva y ética, estas dos últimas con el mismo número de relaciones (3).

TABLA 7. Relaciones externas (resumen codificado).

\begin{tabular}{|c|c|c|}
\hline Dimensión & $\mathbf{S}$ & $\begin{array}{l}\text { Relaciones } \\
\text { externas }\end{array}$ \\
\hline \multirow[t]{3}{*}{ Cognitiva } & S1 & S6, S8 \\
\hline & $\mathrm{S} 2.1$ & $\mathrm{~S} 11$ \\
\hline & $\mathrm{S} 4$ & S9 \\
\hline \multirow[t]{4}{*}{ Metacognitiva } & S9 & S4 \\
\hline & S6 & $\mathrm{S} 1, \mathrm{~S} 10, \mathrm{~S} 12$ \\
\hline & S8 & $\mathrm{S} 1, \mathrm{~S} 7, \mathrm{~S} 15$ \\
\hline & $\mathrm{S} 7$ & $\mathrm{~S} 15$ \\
\hline \multirow{2}{*}{$\begin{array}{l}\text { Afectiva- } \\
\text { Motivacional }\end{array}$} & $\mathrm{S} 10$ & $\mathrm{~S} 17$ \\
\hline & $\mathrm{S} 11$ & $\mathrm{~S} 2.1, \mathrm{~S} 17$ \\
\hline \multirow{3}{*}{$\begin{array}{l}\text { Social/ } \\
\text { Relacional }\end{array}$} & $\mathrm{S} 16$ & S19 \\
\hline & $\mathrm{S} 15$ & S7, S8 \\
\hline & $\mathrm{S} 17$ & $\begin{array}{l}\mathrm{S} 10, \mathrm{~S} 11, \mathrm{~S} 20, \\
\mathrm{~S} 21\end{array}$ \\
\hline \multirow[t]{3}{*}{ Ética } & $\mathrm{S} 19$ & $\mathrm{~S} 16$ \\
\hline & $\mathrm{S} 20$ & S17 \\
\hline & $\mathrm{S} 21$ & $\mathrm{~S} 17$ \\
\hline
\end{tabular}

$\mathrm{S}=$ Subdimensión

Fuente: Elaboración propia. 
GRÁFICo 2. Resultados del procesamiento.

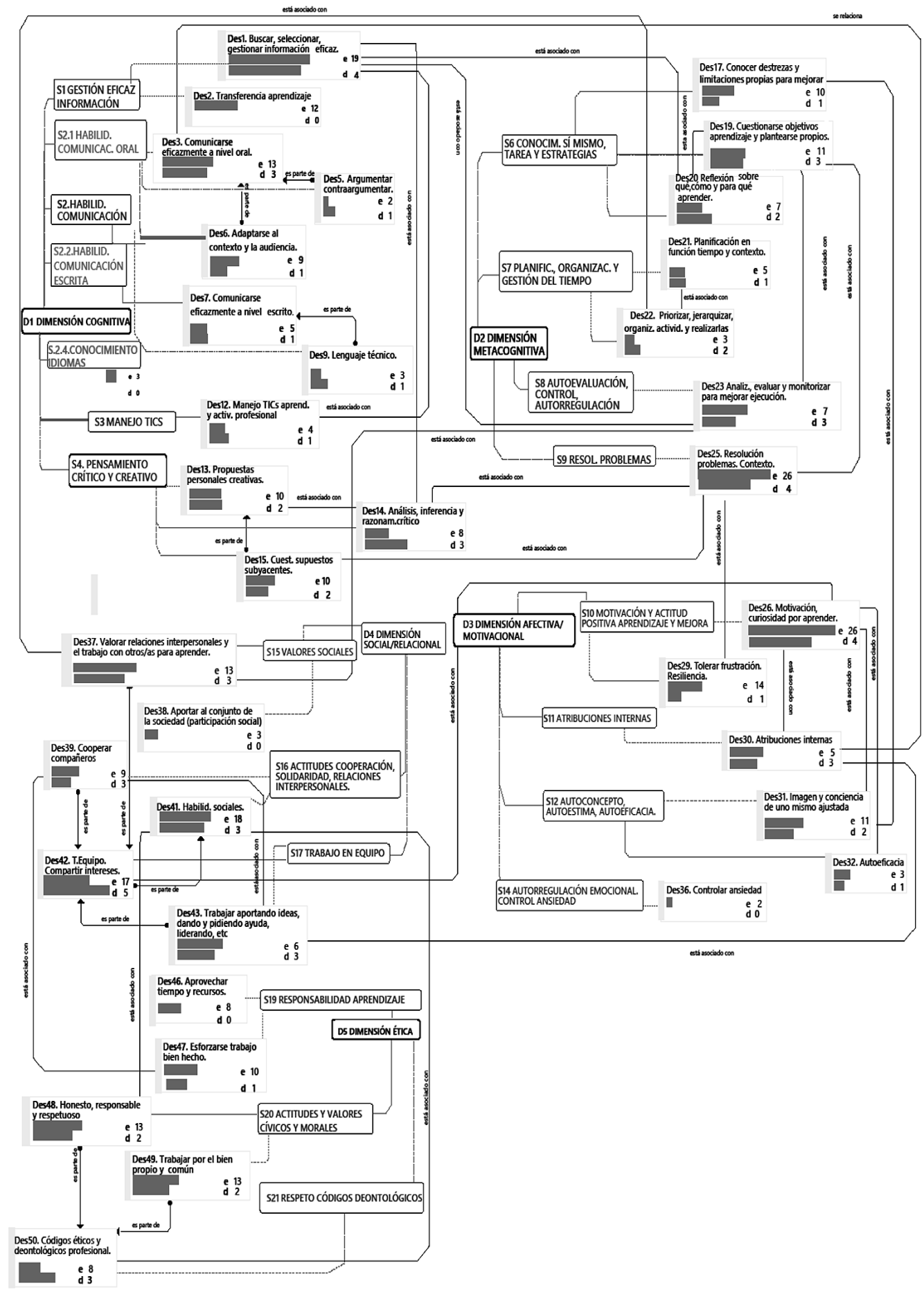

Fuente: Elaboración propia. 


\section{Discusión y conclusiones}

El objetivo del trabajo era contrastar el modelo de competencia AaA del equipo investigador con la valoración de cuatro grupos de informantes clave, considerados como interesados (stakeholders) en el proceso de Bolonia. Tras el análisis de los datos, podemos afirmar que las dimensiones y casi todas las subdimensiones propuestas fueron refrendadas por los participantes.

Desde nuestro punto de vista, esto es sumamente relevante. Por un lado, porque el hecho de que los empleadores, profesionales, profesores y alumnos interpreten que aprender a aprender integra los aspectos nucleares/dimensiones incluidos en el modelo del equipo investigador, sin haber recibido más instrucciones que la pregunta abierta formulada para los grupos, es un importante aval para la validez del modelo. Por otro, porque, habida cuenta de que tanto profesores como alumnos, que comparten el proceso de enseñanza-aprendizaje, consideran importantes los componentes del mismo, esto puede favorecer su inclusión en las propuestas formativas que hay que desarrollar en los currículos de los grados universitarios. Si eso ocurre también, como es el caso, con empleadores y profesionales que saben de las habilidades, actitudes, etc., vinculadas al AaA, necesarias para el trabajo y para la inserción profesional, sobran argumentos para apostar fuerte por la enseñanza de la competencia en la formación universitaria.

Como decíamos arriba, todas las dimensiones aparecieron con claridad en las intervenciones de los participantes, aunque no todas las subdimensiones: dos no aparecieron en las transcripciones (S12, «bienestar físico y emocional» y S17, «control de las condiciones ambientales»). Los participantes aportaron una nueva de «conocimiento de idiomas extranjeros» (S2.4). La decisión final de mantener 0 no estas subdimensiones en el modelo se tomará a partir de su validación estadística.

Dentro de la dimensión cognitiva las cinco subdimensiones aparecen con claridad. Es muy relevante la S1, "gestión eficaz de la información» $(\mathrm{e}=31)$, relativa a la búsqueda, selección y tratamiento de la información. También presenta conexiones con otras de la dimensión cognitiva y metacognitiva, lo que es importante. Otra relevante es la S4, «pensamiento crítico y creativo» $(\mathrm{e}=28)$, conectada con otras cognitivas, metacognitivas y sociales, lo que muestra su importancia y vinculación. También lo es la subdimensión S2.1, «habilidades de comunicación oral» $(\mathrm{e}=24) ; \mathrm{y}$, con menos peso, la S2.2, «habilidades de comunicación escrita» $(e=8)$, al igual que la S4, «manejo de TICs» $(\mathrm{e}=4)$. Ya mencionamos que los participantes incluyeron una nueva subdimensión, "conocimiento de idiomas» $(\mathrm{e}=3$ ). Dentro de la $\mathrm{S} 2$, de «habilidades de comunicación», no hicieron alusión a la S2.3, de «conocimiento y manejo de lenguajes no verbales».

Dentro de la dimensión metacognitiva hay claras referencias también a sus cuatro subdimensiones, siendo especialmente relevante la S6, «conocimiento de sí mismo, de la tarea y de las estrategias» $(\mathrm{e}=35)$, conectada con otras de la dimensión cognitiva y metacognitiva. Es también poderosa la $\mathrm{S} 9$, «resolución de problemas» $(\mathrm{e}=26)$, 
relacionada con códigos cognitivos, metacognitivos y afectivo-motivacionales. Las otras dos subdimensiones, con menor enraizamiento, son la S7, «planificación, organización y gestión del tiempo» $(\mathrm{e}=8)$, y la S8, «autoevaluación, control, autorregulación» $(e=7)$.

Dentro de la dimensión afectivomotivacional aparecen mencionadas cuatro de las cinco subdimensiones. Es especialmente relevante la S10, «motivación y actitud positiva», frente al «aprendizaje», conectada con otras afectivasy sociales/relacionales. También la S12, «autoconcepto, autoestima, autoeficacia» ( $\mathrm{d}=14)$, relacionada con la dimensión metacognitiva y afectiva. Presentan menores frecuencias las subdimensiones S11, «atribuciones internas» $(\mathrm{d}=5)$, relacionada con otras de la dimensión afectiva y relacional, y la S14, «autorregulación emocional y control de la ansiedad» $(\mathrm{d}=5)$.

En la dimensión social/relacional aparecen mencionadas tres de las cuatro subdimensiones, todas importantes, dado su enraizamiento y densidad. La S16, «actitudes de cooperación y solidaridad», es la más mencionada $(e=27)$, seguida de la S17, «trabajo en equipo» $(\mathrm{e}=23)$ y de la S15, «valores sociales» $(e=16)$. Presentan, además, conexiones con otras subdimensiones sociales/relacionales, cognitivas, metacognitivas y afectivas.

En la dimensión ética las tres subdimensiones son relevantes: la S20, «actitudes y valores cívicos y morales», es la más mencionada $(e=26)$, seguida de la S19, «responsabilidad en el aprendizaje» (e=18), y de la S21, «respeto a los códigos éticos y deontológicos» $(\mathrm{e}=8)$. Las dos primeras interaccionan con otras subdimensiones de la dimensión social y las tres con otras de la misma dimensión ética.

\subsection{Un modelo coherente}

Las relaciones internas y externas explicitadas muestran un modelo coherente.

Las relaciones externas muestran que el modelo inicial es consistente al conectarse todas las dimensiones entre sí. Es cierto que la dimensión ética es la menos conectada, tanto externa como internamente. Probablemente, no es cuestión de irrelevancia de los aspectos éticos, sino de falta de conciencia de los participantes. Además, esta dimensión no se contempla en los modelos teóricos previos sobre la competencia AaA (Hautamäki et al., 2002; Hoskins y Friedriksson, 2008; Stringher, 2014), siendo una aportación novedosa de nuestro grupo.

Las relaciones internas establecidas por los participantes en los grupos en la dimensión cognitiva (D1) tienen una clara lógica: «argumentar y contraargumentar» y «adaptarse al contexto y a la audiencia» se integran en «comunicarse eficazmente a nivel oral». "Cuestionar supuestos subyacentes» y llevar a cabo «análisis, inferencia y razonamiento críticos» tienen que ver con la elaboración de «propuestas personales creativas».

Cuando se trató de elementos metacognitivos (D2), la conexión es sumamente coherente: «cuestionarse objetivos de aprendizaje y plantearse objetivos propios» 
guarda relación con la «reflexión sobre qué, cómo y para qué aprender»; con «analizar, evaluar y monitorizar para mejorar la ejecución», y con la «resolución de problemas».

En la dimensión afectiva/motivacional (D3) la «motivación y curiosidad por aprender» se asoció con elementos afectivos y referidos al locus de control, como las «atribuciones internas», la «autoeficacia» y tener una «imagen y conciencia de uno mismo ajustada».

Algo similar ocurrió con la dimensión ética (D5): seguir los «códigos éticos y deontológicos...» estuvo ligado a ser «honesto, responsable y respetuoso» y a «trabajar por el bien propio y común», tal y como marcan dichos códigos, por lo gene- ral, en cualquier ámbito profesional y de conocimiento.

Las conexiones internas más complejas son las de la dimensión social/relacional (D4). Al estar tan conectadas las subdimensiones en términos relativos, resulta difícil establecer agrupaciones en función de los vínculos. Hemos interpretado esto como un indicador de la coherencia interna de esta dimensión, puesto que los participantes relacionaron todas las subdimensiones entre sí, a excepción de una, que fue «aportar al conjunto de la sociedad».

Las dimensiones con más peso relativo de conexión interna después de la social/ relacional fueron la cognitiva y metacognitiva (Gráfico 3).

Gráfico 3. Pesos relativos de conexión interna.

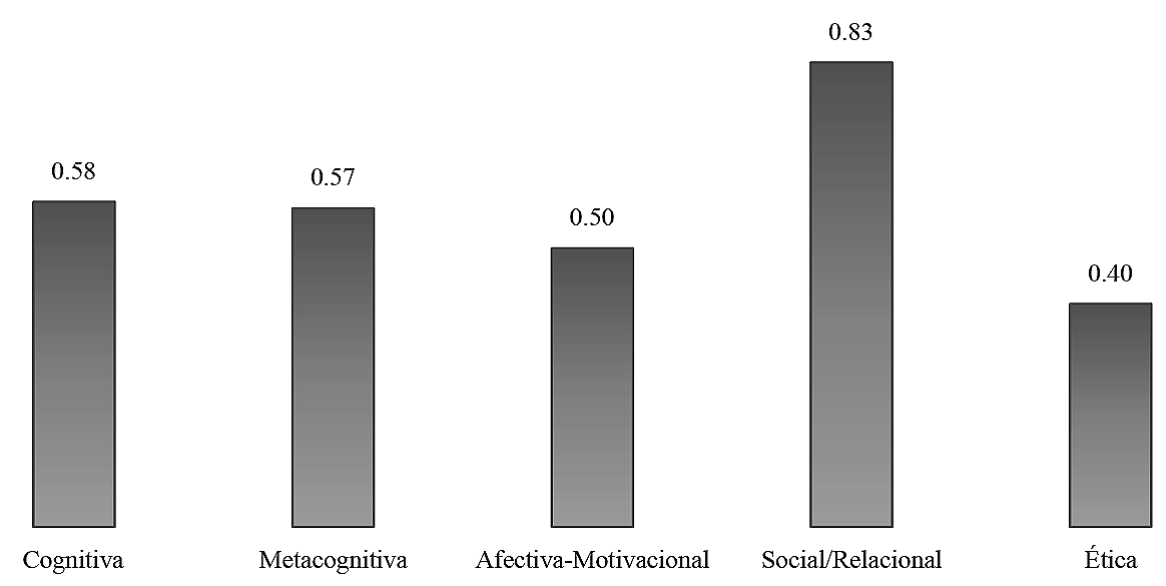

\subsection{Las conexiones de las dimensiones}

Al margen del enraizamiento y la densidad de cada subdimensión, la conexión intradimensional es un indicador de delimitación.
Los participantes atribuyeron una importante conexión interna relativa a las dimensiones que aparecen más tempranamente en el historial científico. Así, la dimensión cognitiva obtuvo un puntaje re- 
lativo intradimensional mayor, seguida de la metacognitiva, la afectivo-motivacional y la ética. Sin embargo, la dimensión social/ relacional fue la más delimitada en función de su conexión interna.

En la literatura las tres primeras aparecen pronto y sus contenidos se mantienen bastante estables, incorporando nuevas aportaciones a partir de las investigaciones (Panadero, 2017; Thoutenhoofd y Pirrie, 2013). En los primeros modelos de aprendizaje estratégico se incluyen tres grandes tipos de estrategias: de procesamiento de la información (cognitivas), de apoyo (afectivo-motivacionales) y metacognitivas (Danserau, 1985; Weinstein y Mayer, 1985; Weinstein, 1987).

Lo mismo ocurre en los modelos de aprendizaje autorregulado. Los primeros trabajos de Zimmerman, fundamentados en la teoría de cognición social de Bandura, incorporaban, en su modelo triádico, aspectos del entorno, de la conducta y de la persona, siempre en la perspectiva del aprendiz que se autorregula en los tres ámbitos. Más tarde, en su modelo cíclico de 2000, definió tres fases, que se mantienen en el de 2009 y en el de 2013 (Zimmerman, 2000, 2013; Zimmerman y Moylan, 2009): la fase de previsión (incluye el análisis de tareas y automotivación), la de ejecución (autoobservación y autocontrol) y la de auto-reflexión (autojuicio y autorreacción). En ellos aparecen evidentes las dimensiones cognitiva, metacognitiva y afectivo-motivacional. También Boekaerts (1996) las incluye en su modelo.

Es verdad que en las investigaciones que articulan el constructo aprendizaje es- tratégico se ve al aprendiz sobre todo como un sujeto individual frente al aprendizaje, hasta cierto punto un "aprendiz aislado", que debe ser capaz de gestionar de modo autónomo sus procesos de aprendizaje. Sin embargo, en la teoría del aprendizaje autorregulado se concede progresivamente más importancia a la construcción del conocimiento en comunidad, en línea de cognición compartida y situada, junto con otros, en contexto y con un planteamiento de metacognición socializada y cooperativa (Thoutenhoofd y Pirrie, 2013), de modo que uno va construyendo sus habilidades metacognitivas y de autorregulación, co-regulación, ayudado por los otros (Allal, 2011). Ello es así por la fundamentación socio-cognitiva de este último constructo, que enfatiza la interacción entre la persona, las habilidades, el contexto y la situación (Panadero, 2017).

También es cierto que los estudios que han servido de referencia para la dimensión social/relacional no siguen un claro patrón de continuidad en la historia, común en las otras dimensiones - salvo la dimensión ética, sin precedentes-. La teoría del aprendizaje cognitivo-social de Bandura (1986) y el enfoque socio-cognitivo en general nos inspiraron para el diseño del modelo teórico, pero también otros estudios que abrieron nuevas líneas acerca de la dimensión social del aprendizaje, sin avanzar necesariamente sobre la base anterior. Así, los trabajos de Kagan (1990), que evidencian la relevancia de aspectos como la cooperación e interdependencia en el proceso de aprendizaje, o los de Shaver (1991), referidos a la adquisición y reformulación de valores sociales. Estos aspec- 
tos también fueron introducidos en el modelo teórico inicial, teniendo claro que en la dimensión social/relacional no hay un punto de inicio claro en el historial de las investigaciones. Hay varios inicios, y no siempre se ha avanzado sobre lo anterior.

La dimensión ética es una aportación de este equipo investigador, como dijimos, y aparece claramente refrendada por los participantes en los grupos, que mencionan 52 veces aspectos incluidos en la dimensión, lo que es sumamente relevante. Un modelo de la competencia AaA no puede prescindir de los aspectos éticos relacionados con el aprendizaje. No deja de sorprender que, a pesar de la progresiva incorporación de modelos como el de aprendizaje servicio, que tiene una clara carga ética (González-Geraldo, Jover y Martínez, 2017), en la formación universitaria, a pesar de trabajos que abordan cómo trabajar en los procesos de aprendizaje las cuestiones éticas (De Reuyter y Schinkel, 2017), o que hacen referencia a la relación entre ciudadanía activa, sus aspectos éticos y aprender a aprender (Hoskins y Deakin Crick, 2010), no sea esta una dimensión contemplada explícitamente en los modelos de la competencia aprender a aprender disponibles hasta el momento. Tenemos que reconocer, no obstante, que en la más reciente formulación de la competencia desarrollada por la CE (2018), sí se hace referencia a elementos éticos: así, se alude a la necesidad de desarrollar actitudes de colaboración y tolerancia y a sentir empatía, respetar a los otros, comprender puntos de vista diferentes, superar prejuicios y comprometerse, actuando con integridad. Sin embargo, tales aspectos aparecen más bien vinculados a aspectos sociales del aprendizaje, no como algo con dimensión propia.

Desde nuestro punto de vista, dar la relevancia que merecen a los aspectos sociales-relacionales, junto con los cognitivos, metacognitivos y afectivo-motivacionales, e incluir la dimensión ética entre las dimensiones esenciales de la competencia contribuye de modo relevante a su enriquecimiento y permite disponer de un modelo comprensivo que puede servir de referente para su trabajo en la formación de los estudiantes, universitarios y no universitarios.

\subsection{Limitaciones y futuras líneas de trabajo}

Sorprende que algunas subdimensiones no aparecieran con la fuerza esperada. El «control de la ansiedad» (dimensión afectivo-motivacional) y «argumentar y contraargumentar» (dimensión cognitiva) obtuvieron un $\mathrm{e}=2$, por ejemplo. Este bajo nivel de enraizamiento deriva, probablemente, de la falta de conciencia de los participantes. 0 quizá de la inmediatez, ya que en los grupos de discusión se exige responder a la cuestión planteada sin que los intervinientes la conozcan previamente y sin un período previo de reflexión sobre la misma. Puede también tener que ver con la propia interpretación de los documentos primarios. Esta es una de las principales limitaciones del uso de Atlas.ti.8.

El software utilizado no realiza automáticamente la interpretación y análisis de los datos, es el equipo investigador quien debe hacerlo. Para paliar los problemas de subjetividad, varios miembros 
del equipo realizaron la tarea analíticointerpretativa de los textos, sin limitarse a realizar operaciones mecánicas y deliberando ante posibles dilemas de codificación.

Sería conveniente complementar los resultados de este estudio con el contraste entre las perspectivas de las cuatro audiencias implicadas. El futuro de la presente investigación pasa por validar el contenido de la competencia con pruebas de fiabilidad y de diferencias entre sujetos. A partir de ahí, se procederá a diseñar un cuestionario de evaluación que recoja el contenido de la competencia para su evaluación, lo que permitirá recopilar información y realizar propuestas curriculares para la enseñanza de la competencia.

\section{Notas}

${ }^{1}$ «Diseño operativo de la competencia "aprender a apren. der" para los grados universitarios, instrumentos de eva. luación y propuestas para la enseñanza». Investigación financiada por el Ministerio de Economía, Industria y Competitividad. Código EDU2017-83284-R. Convocatoria Nacional de Ayudas para la financiación de Proyectos de I+D+i Retos de la Sociedad, 2017.

${ }^{2}$ De las veinte subdimensiones presentes en el modelo, solo dos no aparecen en el gráfico: control de las condicio. nes ambientales (dimensión social/relacional) y bienestar físico y emocional (dimensión afectivo-motivacional). Los participantes no las mencionaron.

${ }^{3}$ La fórmula utilizada para el cálculo es $n / N$, donde $n$ es el número de conexiones o relaciones de una dimensión y $\mathrm{N}$ el número total de componentes de la dimensión. Ello permite obtener un puntaje relativo: en este caso hay siete conexiones internas y once componentes: $7 / 12=.583$.

\section{Referencias bibliográficas}

Allal, L. (2011). Pedagogy, didactics and the coregulation of learning: a perspective from the French-language world of educational research. Research Papers in Education, 26 (3), 329-336.
Bandura. A. (1986). Social Foundations of Thought and Action: A Social Cognitive Theory. Englewood Cliffs, NJ: Prentice-Hall.

Boekaerts, M. (1996). Self-regulated learning at the junction of cognition and motivation. European Psychologist, 1 (2), 100-112.

Brannen, J. (2017). Mixing methods: qualitative and quantitative research. London: Routledge.

Caena, F. (2019). Developing a European Framework for the Personal, Social \& Learning lo Learn Key Competence. Luxemboug: Publications Office of the European Union. Recuperado de https://bit. ly/2vBzK8A (Consultado el 10-10-2019)

Comisión Europea (CE) (2006). Recomendación del Parlamento Europeo y del Consejo de 18 de diciembre de 2006 sobre competencias clave para el aprendizaje permanente. Recuperado de https:// bit.ly/2QNgYmo (Consultado el 26-03-2020).

Comisión Europea (CE) (2018). Anexo de la Propuesta de Recomendación del Consejo relativa a las competencias clave para el aprendizaje permanente. Recuperado de https://goo.gl/ YD9pDw (Consultado el 06-10-2019)

Cortina, A. (2013). ¿Para qué sirve realmente la éti$c a$ ? Barcelona: Paidós.

Creswell, J. W. (2010). Mapping the developing landscape of mixed methods research. En Handbook of mixed methods in social and behavioral research. Second Edition (pp. 45-68). Thousand Oaks, CA: Sage.

Danserau, D. F. (1985). Learning Strategy Research. En H. F. O'Neil (Ed.), Learning Strategies (pp. 209-240). Nueva York: Academic Press.

De Reuyter, D. y Schinkel, A. (2017). Ethics education at the university: from teaching an ethics module to education for the good life. Bordón. Revista de Pedagogía, 69 (4), 125-138. doi: https://doi.org/10.13042/Bordon.2017.690409

Deakin Crick, R., Stringher, C. y Ren, K. (2014). Learning to Learn: International perspectives for theory and practice. London: Routledge.

Denzin, N. y Lincoln, Y. S. (Eds.) (2006). The Sage Handbook of Qualitative Research. Thousand Oaks, CA: Sage.

EI-ESU (2012). Bologna with student eyes 2012. Recuperado de https://www.esu-online.org/wp-content/uploads/2016/07/BWSE2012-online1.pdf (Consultado el 06-10-2019). 
Flavell, J. H. y Wellman, H. M. (1977). Metamemory. En R. V. Kail Jr. y J. W. Hagen (Eds.), Perspectives on the development of memory and cognition (pp. 3-33). Hillsdale: LEA.

Gargallo López, B., Pérez-Pérez, C., García-García, F. J., Giménez Beut, J. A. y Portillo Poblador, N. (2020). La competencia aprender a aprender en la universidad: propuesta de modelo teórico. Educación XX1, 23 (1), 19-44. doi: https://doi.org/10.5944/educxx1.23367

GIPU-EA (2020). Grupo de Investigación en Pedagogía Universitaria y Estrategias de Enseñanza y Aprendizaje. Recuperado de https://gipu. blogs.uv.es/ (Consultado el 26-03-2020)

González, J. y Wagenaar, R. (2006). Tuning Educational Structures in Europe II. La contribución de las universidades al proceso de Bolonia. Bilbao: Universidad de Deusto. Recuperado de http:// www.deusto-publicaciones.es/deusto/pdfs/tuning/ tuning04.pdf (Consultado el 04-10-2019).

González-Geraldo, J. L., Jover, G. y Martínez, M. (2017). La ética del aprendizaje servicio en la universidad: una interpretación desde el pragmatismo. Bordón. Revista de Pedagogía, 69 (4), 63-78. doi: https://doi.org/10.13042/Bordon.2017.690405

Grace, S., Innes, E., Patton, N. y Stockhausen, L. (2017). Ethical experiential learning in medi$\mathrm{cal}$, nursing and allied health education: A narrative view. Nurse Education Today, 51, 23-33.

Grupo de trabajo de Bolonia sobre marcos de cualificaciones (2005). Informe sobre: un marco de cualificaciones para el espacio europeo de educación superior. Recuperado de http://www. unizar.es/eees/doc/Marco_cualificaciones.pdf (Consultado el 03-03-2020)

Hautamäki, J., Arinen, P., Eronen, S., Hautamäki, A., Kupiainen, S., Lindblom, B., Scheinin, P. (2002). Assessing Learning-to-Learn: A Framework. Helsinki: Centre for Educational Assessment, Helsinki University / National Board of Education.

Hautamäki, J. y Kupiainen, S. (2014). Leaning to learn in Finland: Theory and policy, research and practice. En R. Deakin Crick, C. Stringher y K. Ren (Eds.), Learning to learn (pp. 179200). Londres y Nueva York: Routledge.

Hoskins, B. y Deakin Crick, R. (2010). Competences for learning to learn and active citizenship: different currencies or two sides of the same coin? European Journal of Education, 45 (1), 121-137. doi: https://doi.org/10.1111/j.1465-3435.2009.01419.x
Hoskins, B. y Fredriksson, U. (2008). Learning to learn: what is it and can it be measured. Ispra: European Commission. Joint Research Centre, Institute for the Protection and Security of the Citizen. Centre for Research on Lifelong Learning (CRELL).

Jornet Meliá, J. J., García-Bellido, R. y González-Such, J. (2012). Evaluar la competencia aprender a aprender: una propuesta metodológica. Profesorado. Revista de currículum y formación del profesorado, 16 (1), 103-123.

Kagan, S. (1990). Cooperative learning resources for teachers. San Juan de Capistrano, CA: Resources for Teachers.

Kass, M. y Faden, R. R. (2018). Ethics and learning health care: the essentials roles of engagement, transparency, and accountability. Learning Health Systems, 2 (4), 1-3. doi: https://doi. org/10.1002//rh2.10066

Kupiainen, S., Hautamäki, J. y Rantanen, P. (2008). EU pre-pilot on learning to learn: Report on the compiled data, 2008-1190/001-001 TRA-TRINDC. Bruselas: Comisión Europea.

Merriam, S. B. y Grenier, R. S. (Eds.) (2019). Qualitative research in practice: examples for discussion and analysis. San Francisco: Jossey-Bass.

Moreno, A., Cercadillo, L. y Martínez, M. (2008). Learn European Project. Pre-Pilot Study National Report. Madrid: Ministerio de Educación, Cultura y Deporte.

Moreno, A. y Martín, E. (2014). The Spanish approaching to learning to learn. En R. Deakin Crick, C. Stringher y K. Ren, Learning to learn (pp. 196-213). Londres y Nueva York: Routledge.

Muñoz-San Roque, I., Martín-Alonso, J. F., Prieto-Navarro, L. y Urosa-Sanz, B. (2016). Autopercepción del nivel de desarrollo de la competencia de aprender a aprender en el contexto universitario: propuesta de un instrumento de evaluación. Revista de Investigación Educativa, 34 (2), 369-383.

Panadero, E. (2017). A Review of Self-Regulated Learning: Six Models and Four Directions for Research. Frontiers in Psychology, 8 (422), 1-28. Pintrich, P. R. (2004). A conceptual framework for assessing motivation and self-regulated learning in college students. Educational Psychology Review, 16 (4), 385-407. 
Shaver, J. P. (Ed.) (1991). Handbook of research on social studies teaching and learning. New York, NY: Maxwell Macmillan International.

Schmeck, R. R. (1988). Learning strategies and learning styles. Nueva York: Plenum Press.

Stringher, C. (2014). What is learning to learn? A learning to learn process and output model. En R. Deakin Crick, C. Stringher y K. Ren (Eds.), Learning to learn (pp. 9-32). Londres y Nueva York: Routledge.

Thoutenhoofd, E. D. y Pirrie, A. (2013). From self-regulation to learning to learn: observations on the construction of self and learning. British Educational Research Journal, 41 (1), 72-84.

Villardón-Gallego, L., Yániz, C., Achurra, C., Iraurgi, I. y Aguilar, M. C. (2013). Learning competence in university: development and structural validation of a scale to measure. Psicodidáctica, 18 (2), 357-374.

Weinstein, C. E. (1987). LASSI User's Manual. Clearwater, FL: H\&H and Publishing Company.

Weinstein, C. E. (1988). Assessment and training of student learning strategies. En R. R. Schmeck (Ed.), Learning strategies and learning styles (pp. 291-316). Nueva York: Plenum Press.

Weinstein, C. E., Husman, J. y Dierking, D. (2002). Self-Regulation Interventions with a focus on learning strategies. En M. Boekaerts, P. R. Pintrich y M. Zeinder, Handbook of Self-regulation (pp. 727-747). San Diego: Academic Press.

Weinstein, C. E. y Mayer, R. E. (1985). The teaching of learning strategies. En M. C. Wittrock, (Ed.), Handbook of research on teaching (pp. 315-327). Nueva York: MacMillan.

Wolf, N. H. y Silver, Ch. (2017). Qualitative analysis using Atlas.ti. Nueva York: Routledge.

Yip, M. C. W. (2012). Learning strategies and self-efficacy as predictors of academic performance: a preliminary study. Quality in Higher Education, 18 (1), 23-34.

Zimmerman, B. J. (2000). Attaining self-regulation: a social cognitive perspective. En M. Boekaerts, P. R. Pintrich y M. Zeidner (Eds.), Handbook of Self-Regulation (pp. 13-40). San Diego, CA: Academic Press.

Zimmerman, B. J. (2013). From cognitive modeling to self-regulation: a social cognitive career path. Educational Psychologist, 48, 135-147.
Zimmerman, B. J. y Moylan, A. R. (2009). Self-regulation: where metacognition and motivation intersect. En J. Hacker, J. Dunlosky y A. C. Graesser (eds.), Handbook of Metacognition in Education (pp. 299-315). Nueva York: Routledge.

\section{Biografía de los autores}

Bernardo Gargallo López es Doctor en Filosofía y Ciencias de la Educación por la Universidad de Valencia y Catedrático de Teoría de la Educación en esta universidad. Primer Premio Nacional de Investigación Educativa en 2000 y en 2002. Su línea de investigación actual y los proyectos que ha dirigido se centran en la enseñanza y el aprendizaje en la universidad. Dirige el grupo GIPU-EA.

(iD https://orcid.org/0000-0002-2805-4129

Fran J. García-García es Personal Investigador en Formación en el Departamento de Teoría de la Educación de la Universidad de Valencia, con la Ayuda de Formación del Profesorado Universitario (FPU) del Ministerio de Educación, Cultura y Deporte (FPU17/00156). Ganó el Premio Extraordinario de Máster en Educación Especial. Sus últimos trabajos versan sobre la pedagogía universitaria y el aprendizaje de los estudiantes en educación superior.

(iD https://orcid.org/0000-0002-6267-0080

Inmaculada López Francés es Doctora en Educación por la Universidad de Valencia y Profesora Ayudante Doctora. Premio de Licenciatura y Doctorado por la Universidad de Valencia. Entre sus líneas de investigación encontramos la diversidad sexual y afectiva, identidad y género y pedagogía universitaria.

(iD https://orcid.org/0000-0003-1178-9054 
Miguel Ángel Jiménez Rodríguez es Doctor en Filosofía y Ciencias de la Educación por la Universidad de Valencia. Premio Extraordinario de Licenciatura. Profesor de la Universidad Católica de Valencia. Ha sido Vicedecano de Psicopedagogía, Magisterio y Educación Social. Director del centro de formación continua Educa-Acción de la misma universidad. Miembro del equipo GIPU-EA. Tiene una dilatada trayectoria investigadora en el campo de las competencias.

iD https://orcid.org/0000-0002-1550-6157
María Salomé Moreno Navarro es Arquitecta y Especialista Universitaria en Pedagogía Universitaria por la Universidad Politécnica de Valencia, en la que es Profesora Asociada. Colabora en proyectos competitivos con el grupo GIPU-EA y ha participado en dos proyectos PIME de la Universidad Politécnica de Valencia.

(iD https://orcid.org/0000-0003-4578-5959 


\section{revista española de pedagogía año 78, n. ${ }^{\circ} 276$, mayo-agosto 2020 \\ Spanish Journal of Pedagogy \\ year 78, n. 276, May-August 2020 \\ Sumario* \\ Table of Contents **}

\section{Editorial \\ Editorial}

José Antonio Ibáñez-Martín

Una consideración educativa sobre la pandemia:

resistir... y adelantar

An educational consideration on the pandemic: endure...

and progress

\section{Estudios \\ Studies}

Bernardo Gargallo López, Fran J. García-García, Inmaculada López-Francés,

Miguel Ángel Jiménez Rodriguez y

Salomé Moreno Navarro

La competencia aprender a aprender:

valoración de un modelo teórico

The learning to learn competence: An assessment of a theoretical model

Miguel A. Santos Rego, María José Ferraces Otero, İor Mella Núñez y Ana Vázquez-Rodríguez

Universidad, competencias cívico-sociales

y mercado de trabajo

University, civic-social competences,

and the labour market
Jesús Miguel Jornet Meliá, María Jesús Perales

Montolío y José González-Such

El concepto de validez de los procesos

de evaluación de la docencia

The concept of validity of teaching evaluation processes 233

\section{Fernando Acevedo Calamet}

181 Factores explicativos del abandono de los estudios

en la educación superior en contextos socio.

académicos desfavorables

Explanatory factors for dropout from higher education in unfavourable socio-academic contexts

\section{Notas \\ Notes}

Maria-Carmen Ricoy y Cristina Sánchez-Martínez

Revisión sistemática sobre el uso de la tableta en la etapa de educación primaria

A systematic review oftablet use in primary education

Jaime Carcamo-Oyarzun y Christian Herrmann

Validez de constructo de la batería MOBAK para la evaluación de las competencias motrices básicas en escolares de educación primaria

Construct validity of the MOBAK test battery for the assessment

\footnotetext{
* Todos los artículos están también publicados en inglés en la página web de la revista: https://revistadepedagogia.org.

** All the articles are also published in English on the web page of the journal: https://revistadepedagogia.org.
} 
Concha Iriarte Redin, Sara Ibarrola-García y Maite Aznárez-Sanado

Propuesta de un instrumento de evaluación de la mediación escolar (CEM)

Proposal for a school mediation evaluation tool (MEQ)

309

\section{Bruno Echauri Galván y Silvia García Hernández}

Traducir en colores: la traducción como herramienta de evaluación de la comprensión lectora en inglés como lengua extranjera

Translating in colours: Translation as an assessment tool for reading comprehension in English as a second language

\section{Reseñas bibliográficas}

Esteban-Bara, F. (2019). La universidad light: Un análisis de nuestra formación universitaria (José L. González-Geraldo). Ruiz-Corbella, M. y GarcíaGutiérrez, J. (Eds.) (2019). Aprendizaje-Servicio. Los retos de la evaluación (Andrea Muñoz Villanueva). Lafforgue, L. (2019). Recuperemos la escuela (Beatriz Gálvez). Ferraces-Otero, M. J., Godás-Otero, A. y García-Álvarez, J. (2019). Cómo realizar un estudio científico en ciencias sociales, de la educación y de la salud (Carolina Rodríguez-Llorente).

\section{Informaciones}

46. ${ }^{a}$ conferencia anual de la Association for Moral

327 Education sobre el tema: «Morality, Environmental Sustainability, and Education»; XV Congreso Internacional de Teoría de la Educación: «Democracia y tradición en la teoría y la práctica educativa del siglo XXI. En el 50 aniversario de la Ley General de Educacióny.

\section{Instrucciones para los autores}

Instructions for authors 365

ISSN: 0034-9461 (Impreso), 2174-0909 (Online)

https://revistadepedagogia.org/

Depósito legal: M. 6.020 - 1958

INDUSTRIA GRÁFICA ANZOS, S.L. Fuenlabrada - Madrid 


\title{
The learning to learn competence: An assessment of a theoretical model La competencia aprender a aprender: valoración de un modelo teórico
}

\begin{abstract}
Bernardo GARGALLO LÓPEZ, PhD. Professor. Universidad de Valencia (bernardo.gargallo@uv.es). Fran J. GARCÍA-GARCÍA. Research Trainee. Universidad de Valencia (Francisco.Javier.Garcia-Garcia@uv.es). Inmaculada LÓPEZ-FRANCÉS, PhD. Assistant Professor. Universidad de Valencia (Inmaculada.Lopez-Frances@uv.es). Miguel Ángel JIMÉNEZ RODRÍGUEZ, PhD. Associate Professor. Universidad Católica de Valencia (mangel.jimenez@ucv.es). Salomé MORENO NAVARRO. Professor of Practice. Universidad Politécnica de Valencia (smoreno@upvnet.upv.es).
\end{abstract}

\section{Abstract:}

Learning to learn (LTL) is a key competence (European Commission [EC], 2006, 2018). Building it into the educational system requires a solid theoretical model that researchers share, but there is currently a lack of agreement among academics. This work aims to check the theoretical model proposed by the research team against the opinions of key informants. This model has five dimensions - cognitive, metacognitive, affective-motivational, social-relational, andethical — aswellastwentysubdimensions. The first three dimensions come from the literature about strategic and self-regulated learning and have been present since research into this topic began. The fourth comes from the social-cognitive approach and has more recently been added into explanatory models. The fifth one is an original contribution by this group. Our research focusses on the university setting and the aim of the present work is to verify our model with some of the key informants involved in the process (students, teachers, professionals, and employers). A qualitative methodology was used, featuring twelve discussion groups, one for each group in the three participating universities $(\mathrm{N}=67)$. The participants were asked a general question about LTL and then their contributions were recorded. These contributions were then transcribed and processed using Atlas ti.8. to compare them with the theoretical model. The presence and importance of established dimensions and subdimensions was analysed, considering the frequency

Revision accepted: 2020-03-04.

This is the English version of an article originally printed in Spanish in issue 276 of the revista española de pedagogía. For this reason, the abbreviation EV has been added to the page numbers. Please, cite this article as follows: Gargallo López, B., García-García, F. J., López-Francés, I., Jiménez Rodríguez, M. Á., \& Moreno Navarro, S. (2020). La competencia aprender a aprender: valoración de un modelo teórico | The learning to learn competence: An assessment of a theoretical model. Revista Española de Pedagogía, 78 (276), 187-211. doi: https://doi.org/10.22550/REP78-2-2020-05 https://revistadepedagogia.org/ ISSN: 0034-9461 (Print), 2174-0909 (Online) 
of comments and their connections and interrelationships. The results corroborated the model proposed by the research team: all the dimensions and almost all of the subdimensions appeared in the participants' comments. The next stage in the research process is to develop a standardised assessment instrument, based on the model, so it can be statistically validated with university students.

Keywords: key competences, learning to learn competence, university students, strategic learning, self-regulated learning.

\section{Resumen:}

La competencia aprender a aprender (AaA) es una competencia clave (Comisión Europea [CE], 2006, 2018). Su incorporación en el sistema educativo precisa de un modelo teórico sólido y compartido por los investigadores, pero no hay acuerdo suficiente entre los académicos. Este trabajo pretende contrastar el modelo teórico elaborado por el equipo investigador con la opinión de informantes clave. Este modelo integra cinco dimensiones —cognitiva, metacognitiva, afectivo-motivacional, social-relacional y éticay veinte subdimensiones. Las tres primeras provienen de la literatura sobre aprendizaje estratégico y autorregulado y están presentes desde los inicios de la investigación sobre el tema. La cuarta proviene del enfoque socio-cognitivo y se incorporó más tarde a los modelos explicativos. La quinta es una aportación original de este grupo. Nuestro contexto de investigación es la universidad y el objetivo contrastar nuestro modelo con informantes clave involucrados en el proceso (estudiantes, profesores, profesionales y empleadores). Para ello, se utilizó metodología cualitativa, realizándose doce grupos de discusión, uno por cada colectivo, en las tres universidades participantes en el proyecto $(\mathrm{N}=67)$. A partir de una pregunta general sobre lo que suponía la competencia AaA para los participantes, sus aportaciones fueron grabadas, transcritas y procesadas con Atlas ti.8. para cotejarlas con el modelo teórico. Se analizaron la presencia y relevancia de las dimensiones y subdimensiones establecidas, prestando atención a la frecuencia de los comentarios y a sus conexiones e interrelaciones. Los resultados corroboraron el modelo propuesto por el equipo investigador: todas las dimensiones y casi todas las subdimensiones aparecieron en las intervenciones de los participantes. La siguiente fase del proceso de investigación es la construcción de un instrumento de evaluación estandarizado, a partir del modelo, para su validación estadística en estudiantes universitarios.

Descriptores: competencias clave, competencia aprender a aprender, estudiantes universitarios, aprendizaje estratégico, aprendizaje autorregulado.

\section{Introduction}

Learning to Learn (LTL) is one of the key competences for the educational system of the member states of the EC (2006). In 2018 the Commission reformulated its proposal (EC, 2018), adding some ethical aspects among other ones to the previous model of what the competence from 2006.

On the basis of the initial recommendation, states have incorporated these key competences into their educational systems. The 
EU's goal is for students to know how to learn when they complete their compulsory education, even though this is more of an aspiration than a reality, and so learning to learn is still fundamental for university-level studies (EI-ESU, 2012). This is also the view of the European Higher Education Area (EHEA).

\subsection{The background to the competence}

The EC's proposals are based on academic literature, the construct of strategic learning (Danserau, 1985; Weinstein, 1987, 1988; Weinstein \& Mayer, 1985), and the construct of self-regulated learning, which developed at the same time, the former being based on cognitive psychology and the information processing theory and the latter on the socio-cognitive focus (Caena, 2019; Thoutenhoofd \& Pirrie, 2013).

Three dimensions have generally been established to delimit the construct of strategic and self-regulated learning: a cognitive dimension (handling skills, techniques and strategies for processing information), a metacognitive one (conscience/control/ self-regulation of one's own learning processes), and an affective-motivational (motivational/attitudinal/affective components that activate and support learning) (Hoskins \& Fredriksson, 2008; Yip, 2012).

\subsection{Research problem}

Evaluating the LTL competence is a key topic in the studies published. This makes sense as working on this competence requires good instruments for evaluating how well it is being achieved. In the case of Spain, the works of Jornet Meliá, García-Bellido, and González-Such, (2012), Muñoz-San Roque, Martín-Alonso, Prieto-Navarro, and
Urosa-Sanz (2016), and Villardón-Gallego, Yániz, Achurra, Iraurgi, and Aguilar (2013), among others, are worth mentioning. All of them focus on evaluation, considering the two most recent standardised instruments for doing this.

There are important works from elsewhere in Europe, such as that of Hautamäki et al. (2002), which aims to construct a framework for evaluation, and that of Hoskins and Fredriksson (2008), who coordinated the work of the CRELL (Center for Research on Education and Lifelong Learning) network to try to reach a consensus on a theoretical model and an evaluation protocol. The results of this network's efforts did not satisfy the researchers, who were from a variety of teams from different EU countries, and it was not possible to reach an agreement on a common European indicator for the competence, with the matter being left open (Kupiainen, Hautamäki, \& Rantanen, 2018; Moreno, Cercadillo, \& Martínez, 2008).

In fact, the most recently published works reflect considerable disagreement among academics with regards to what this competence entails (Deakin Crick, Stringher, \& Ren, 2014).

There is insufficient clarity in the theoretical apparatus of the LTL competence, in its evaluation, and especially in regards to work on it in the university context. Therefore, we intend to develop a solid theoretical model, based on a study of academic literature, as part of the R\&D\&I project we are developing ${ }^{1}$. The research is organised around different benchmarks; 
in this text, we intend to test our model against valuations by the key informants who make up the working groups that follow-up the Bologna Process. We intend to adapt the model gradually in order to determine the curriculum design of this competence in university programmes.

\subsection{The GIPU-EA group's model}

To complete this curriculum design, it is necessary to build a good model of the competence that makes it possible to define what a command of it means (Jornet Meliá et al., 2012), incorporating the components recognised in the scientific community.

To this end, the GIPU-EA Research Group (University Pedagogy and Teaching and Learning Strategies Research Group) reviewed the literature on this topic and organised the theoretical model into three initial dimensions: cognitive, metacognitive, and affective-motivational (Hautamäki et al., 2002; Hoskins \& Fredriksson, 2008; Stringher, 2014; Weinstein, Husman, \& Dierking, 2002; Yip, 2012). All three are clearly present in the theory of learning strategies (Schmeck, 1988; Weinstein, 1987; Weinstein \& Mayer, 1985) and the theory of self-regulated learning (Panadero, 2017; Pintrich, 2004; Zimmerman, 2000), which support the formulation of the learning to learn competence.

A fourth social-relational dimension of learning in context, deriving from the socio-cognitive focus (Panadero, 2017; Stringher, 2014; Thoutenhoofd \& Pirrie, 2013) was incorporated. This reflects the importance of learning with others and from others, along the same lines as situated cognition, as well as improving the context and society through one's own learning. Stringher (2014), in her model, considers the social dimension as significant on the same lines as what we propose: not just comprehension and control of the contextual conditions of tasks, but also incorporating social values, interpersonal relationships, learning with others, etc. Hautamäki et al. (2002) also regard it as a fundamental element of LTL.

Up to this point, our model is consistent with previous ones. However, we believe that the model should be more exacting and we include a fifth ethical dimension relating to learning, which researchers have not previously considered. A competent learner who has «learnt to learn» cannot disregard ethical components, either those relating to ethics in the process of learning or those in the process of using what has been learnt to improve oneself and others (Cortina, 2013; Grace, Innes, Patton, \& Stockhusen, 2017; Kass \& Faden, 2018). The EC is responsive to this position and in the 2018 text it included references to the ethical dimension, as we mentioned above.

The proposed subdimensions of the competence are based on contributions from the literature (Flavell \& Welman, 1977; Hautamäki et al., 2002; Hautamäki \& Kupiainen, 2014; Hoskins \& Fredriksson, 2008; Moreno \& Martín, 2014; Stringher, 2014; Pintrich, 2004; Weinstein et al., 2002; Yip, 2012; Zimmerman, 2000) and based on the research team's own reflections.

A detailed description of the model and of its construction can be found in Gargallo 
López, Pérez-Pérez, García-García, Giménez Beut, \& Portillo Poblador (2020). We propose defining the LTL competence as follows:

Learning to learn involves the ability to organise and regulate one's own learning increasingly effectively and independently depending on one's own objectives, context, and needs. This competence makes it possible to acquire new knowledge, skills, and attitudes, and to solve problems by skilfully applying solutions in a variety of contexts, at personal and professional levels, individually or in a group. It involves being able to learn with others and cooperate effectively to carry out joint tasks. It also involves awareness of one's own skills and limitations and the ability to plan one's own learning tasks effectively, handling the necessary resources, techniques, skills, and learning strategies. It also entails the capacity to self-evaluate and self-regulate one's performance at a cognitive and affective level, optimising capacities in order to overcome obstacles and limitations. Similarly, it requires intellectual curiosity, intrinsic motivation, and an interest in confronting difficulties and overcoming them successfully, maintaining an ethical commitment and a positive attitude to learning.

The graph and tables below include the descriptionofthecompetence:Graph1shows the dimensions proposed with descriptions of them. Tables 1 to 5 show the 20 component subdimensions (S) and give descriptions of them (Des) (50 descriptions).

GRAPH 1. Dimensions of the LTL competence.

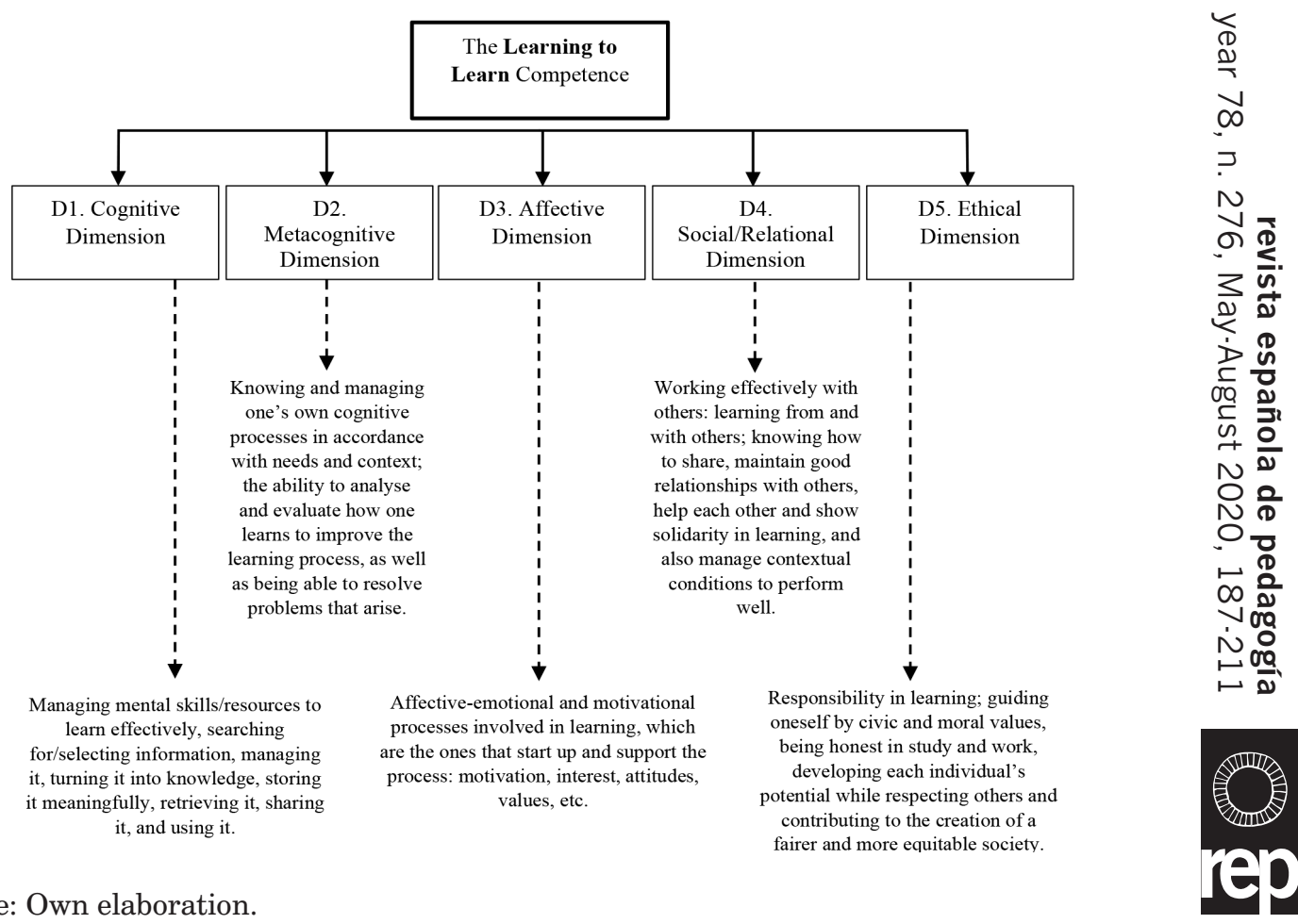

Source: Own elaboration. 
TABLE 1. Subdimensions of the cognitive dimension.

\begin{tabular}{|c|c|c|}
\hline \multicolumn{3}{|c|}{ COGNITIVE DIMENSION } \\
\hline Subdimensions & & Descriptions/Components \\
\hline \multirow{2}{*}{$\begin{array}{l}\text { S1. Managing } \\
\text { information } \\
\text { effectively }\end{array}$} & & $\begin{array}{l}\text { Des1. Using relevant sources of information. Finding and } \\
\text { selecting information, managing it appropriately, turning it into } \\
\text { knowledge, storing it in an understandable way, and retrieving it } \\
\text { effectively to use it. }\end{array}$ \\
\hline & & $\begin{array}{l}\text { Des2. Using and transferring what you have learnt to other } \\
\text { contexts (everyday life, solving problems in the sphere of study } \\
\text { and the professional sphere, etc.). }\end{array}$ \\
\hline \multirow{9}{*}{$\begin{array}{l}\text { S2. Communica- } \\
\text { tion skills }\end{array}$} & \multirow[t]{4}{*}{$\begin{array}{l}\text { S2.1. Oral communi- } \\
\text { cation skills }\end{array}$} & $\begin{array}{l}\text { Des3. Effective oral communication, transmitting knowledge and } \\
\text { expressing ideas clearly, rigorously, and convincingly. }\end{array}$ \\
\hline & & $\begin{array}{l}\text { Des4. Expressing oneself orally in a structured and intelligible } \\
\text { way both in short oral presentations and in long presentations } \\
\text { with subsequent discussion. }\end{array}$ \\
\hline & & Des5. Arguing and counterarguing appropriately. \\
\hline & & Des6. Adapting to the setting and audience. \\
\hline & \multirow[t]{3}{*}{$\begin{array}{l}\text { S2.2. Written com- } \\
\text { munication skills }\end{array}$} & $\begin{array}{l}\text { Des7. Effective written communication, transmitting } \\
\text { knowledge and expressing ideas clearly, rigorously, } \\
\text { and convincingly. }\end{array}$ \\
\hline & & $\begin{array}{l}\text { Des8. Expressing oneself in writing with a logical order and good } \\
\text { construction, preparing well-structured documents tailored to } \\
\text { different purposes. }\end{array}$ \\
\hline & & $\begin{array}{l}\text { Des9. Writing rigorous texts, with a degree of scientific and te- } \\
\text { chnical quality, relating to the area of study, reports, final degree } \\
\text { projects, etc. }\end{array}$ \\
\hline & $\begin{array}{l}\text { S2.3. Knowledge and } \\
\text { use of non-verbal } \\
\text { language }\end{array}$ & $\begin{array}{l}\text { Des10. Knowing and effectively managing non-verbal langua- } \\
\text { ges — visual, iconic, artistic, etc. - insofar as they are used as a } \\
\text { vehicle for learning. }\end{array}$ \\
\hline & $\begin{array}{l}\text { S2.4. Knowing } \\
\text { and using foreign } \\
\text { languages }\end{array}$ & $\begin{array}{l}\text { Des11. Using foreign languages to communicate fluently and for } \\
\text { learning. }\end{array}$ \\
\hline $\begin{array}{l}\text { S3. Using digital } \\
\text { technologies }\end{array}$ & & $\begin{array}{l}\text { Des12. Effectively applying digital technologies to learning and } \\
\text { professional activities (word processing, spreadsheets, presen- } \\
\text { tation software, statistical packages in degrees where they are } \\
\text { necessary, search engines, and databases relating to one's studies } \\
\text { and profession, etc.). }\end{array}$ \\
\hline \multirow{4}{*}{$\begin{array}{l}\text { S4. Critical } \\
\text { and creative } \\
\text { thinking }\end{array}$} & & $\begin{array}{l}\text { Des13. Drawing up creative personal proposals that go beyond } \\
\text { what is given. }\end{array}$ \\
\hline & & $\begin{array}{l}\text { Des14. Carrying out analyses, inferences, and critical reasoning } \\
\text { differentiated by tasks and situations in life. }\end{array}$ \\
\hline & & $\begin{array}{l}\text { Des15. Questioning underlying assumptions in habitual ways of } \\
\text { thinking and acting. }\end{array}$ \\
\hline & & $\begin{array}{l}\text { Des16. Producing new thinking - thinking differently - about } \\
\text { the reality one inhabits. }\end{array}$ \\
\hline
\end{tabular}

Source: Own elaboration. 
Table 2. Subdimensions of the Metacognitive Dimension.

\begin{tabular}{|c|c|}
\hline \multicolumn{2}{|r|}{ METACOGNITIVE DIMENSION } \\
\hline Subdimensions & Descriptions/Components \\
\hline \multirow{4}{*}{$\begin{array}{l}\text { S5. Knowledge of } \\
\text { oneself, of the } \\
\text { task, and of } \\
\text { strategies for } \\
\text { approaching it }\end{array}$} & Des17. Identifying one's own skills and limitations to be able to improve them insofar as it is possible. \\
\hline & $\begin{array}{l}\text { Des18. Thinking about the task and analysing it, the objectives, and the strategies needed to } \\
\text { approach and resolve it. }\end{array}$ \\
\hline & Des19. Questioning learning objectives and setting your own objectives. \\
\hline & $\begin{array}{l}\text { Des20. Taking decisions about what and how to learn, in accordance with one's own objectives } \\
\text { and needs and/or those of one's professional performance. }\end{array}$ \\
\hline \multirow{2}{*}{$\begin{array}{l}\text { S6. Planning, } \\
\text { organising, and } \\
\text { managing time }\end{array}$} & $\begin{array}{l}\text { Des21. Carrying out appropriate planning of asks to achieve the aims planned for the short, } \\
\text { medium, and long term, depending on the context and the time available. }\end{array}$ \\
\hline & Des22. Prioritising, hierarchising, and organising necessary activities and performing them. \\
\hline \multirow[t]{2}{*}{$\begin{array}{l}\text { S7. Self-evaluation, } \\
\text { control, self- } \\
\text { regulation }\end{array}$} & $\begin{array}{l}\text { Des23. Analysing, evaluating, and monitoring one's own performance, establishing the necessary } \\
\text { mechanisms to improve performance and making the necessary changes in planning and imple- } \\
\text { mentation by using more effective strategies and skills. }\end{array}$ \\
\hline & Des24. Seeking guidance, advice, and support if necessary. \\
\hline $\begin{array}{l}\text { S8. Problem } \\
\text { solving }\end{array}$ & $\begin{array}{l}\text { Des } 25 \text {. Analysing and solving problems effectively and in context, identifying and defining the } \\
\text { significant elements of them, developing high-level complex reasoning processes, not just associa- } \\
\text { tive and routine actions. }\end{array}$ \\
\hline
\end{tabular}

Source: Own elaboration.

TABLE 3. Subdimensions of the affective/motivational dimension.

\begin{tabular}{|c|c|}
\hline \multicolumn{2}{|r|}{ THE AFFECTIVE AND MOTIVATIONAL DIMENSION } \\
\hline Subdimensions & Descriptions/Components \\
\hline \multirow{4}{*}{$\begin{array}{l}\text { S9. Motivation } \\
\text { and positive } \\
\text { attitude } \\
\text { towards } \\
\text { learning and } \\
\text { improvement }\end{array}$} & $\begin{array}{l}\text { Des26. Developing and maintaining motivation, curiosity, interest, and taste for understanding } \\
\text { content and in-depth learning. }\end{array}$ \\
\hline & $\begin{array}{l}\text { Des27. Developing a firm will to learn, guiding action in the right direction to achieve good } \\
\text { results. }\end{array}$ \\
\hline & Des28. Perseverance in learning, concentrating for extended periods of time, overcoming difficulties. \\
\hline & Des29. Tolerating frustration when you do not succeed in learning. Resilience. \\
\hline $\begin{array}{l}\text { S10. Internal } \\
\text { attribution }\end{array}$ & $\begin{array}{l}\text { Des30. Attributing learning outcomes and performance to one's own efforts, which one can control } \\
\text { and manage. }\end{array}$ \\
\hline \multirow{2}{*}{$\begin{array}{l}\text { S11. Self-concept } \\
\text { and self-es- } \\
\text { teem Self-effi- } \\
\text { cacy }\end{array}$} & $\begin{array}{l}\text { Des31. Having an accurate self-image, which involves accepting and appreciating oneself and is } \\
\text { compatible with awareness of one's own limitations and with efforts to improve. }\end{array}$ \\
\hline & $\begin{array}{l}\text { Des32. Increasing self-efficacy, feeling capable of achieving demanding objectives, and of succeeding } \\
\text { in performing tasks. }\end{array}$ \\
\hline \multirow{2}{*}{$\begin{array}{l}\text { S12. Physical and } \\
\text { emotional } \\
\text { well-being }\end{array}$} & Des33. Maintaining good physical and emotional condition, suitable for learning and working. \\
\hline & Des34. Leading a healthy life (diet, rest, sleep, and exercise) that helps with learning. \\
\hline \multirow{2}{*}{$\begin{array}{l}\text { S13. Emotional } \\
\text { self-regulation } \\
\text { and contro- } \\
\text { lling anxiety }\end{array}$} & $\begin{array}{l}\text { Des35. Observing, analysing, and modifying emotional reactions in a socially acceptable way depen- } \\
\text { ding on learning objectives. }\end{array}$ \\
\hline & $\begin{array}{l}\text { Des36. Controlling anxiety, learning to relax in stressful situations (exams, presentations, public } \\
\text { speeches, etc.). }\end{array}$ \\
\hline
\end{tabular}

Source: Own elaboration. 
TABLE 4. Subdimensions of the social-relational dimension.

\begin{tabular}{|c|l|}
\hline \multicolumn{2}{|c|}{ SOCIAL/RELATIONAL DIMENSION } \\
\hline Subdimensions & \multicolumn{1}{|c|}{ Descriptions/Components } \\
\hline S14. Social values & $\begin{array}{l}\text { Des37. Valuing interpersonal relationships and working with others to learn with and } \\
\text { from them. }\end{array}$ \\
\cline { 2 - 3 } & $\begin{array}{l}\text { Des38. Working, studying, and making an effort to contribute to society as a whole, not just for } \\
\text { one's own personal development. }\end{array}$ \\
\hline $\begin{array}{c}\text { S15. Attitudes of } \\
\text { cooperation } \\
\text { and solidarity; } \\
\text { interpersonal } \\
\text { relationships }\end{array}$ & $\begin{array}{l}\text { Des39. Cooperating effectively with classmates to solve problems, study, learn with and from } \\
\text { them, and work. }\end{array}$ \\
\cline { 2 - 3 } $\begin{array}{l}\text { Des40. Establishing and maintaining good interpersonal relationships with classmates and } \\
\text { teachers. }\end{array}$ \\
\cline { 2 - 3 } $\begin{array}{l}\text { S16. Team work } \\
\text { social skills: listening, empathy, assertiveness, and solidarity in relationships with others. }\end{array}$ \\
\hline $\begin{array}{l}\text { Des42. Carrying out tasks with shared objectives and interests, overcoming difficulties that } \\
\text { arise when doing so. }\end{array}$ \\
\cline { 2 - 3 } $\begin{array}{l}\text { Des43. Participating actively in working groups contributing ideas and efforts, receiving and } \\
\text { giving help, leading when necessary, etc. }\end{array}$ \\
\hline \multirow{2}{*}{$\begin{array}{l}\text { S17. Controlling } \\
\text { environmental } \\
\text { conditions }\end{array}$} & $\begin{array}{l}\text { Des44. Creating an appropriate environment for working and performing that enables concen- } \\
\text { tration and has the necessary measures and resources. }\end{array}$ \\
\cline { 2 - 3 } & Des45. Adjusting the elements in your context to learn better. \\
\hline
\end{tabular}

Source: Own elaboration.

TABLE 5. Subdimensions of the ethical dimension.

\begin{tabular}{|c|l|}
\hline \multicolumn{2}{|c|}{ ETHICAL DIMENSION } \\
\hline Subdimensions & \multicolumn{1}{|c|}{ Descriptions/Components } \\
\hline $\begin{array}{c}\text { S18. Responsibility in } \\
\text { learning }\end{array}$ & $\begin{array}{l}\text { Des46. Maintaining a responsible attitude towards learning, making good use of the available } \\
\text { time and resources. }\end{array}$ \\
\cline { 2 - 2 } & $\begin{array}{l}\text { Des47. Striving for effective and committed work, to do things as well as possible, avoiding } \\
\text { incomplete or badly done work. }\end{array}$ \\
\hline $\begin{array}{c}\text { S19. Civic and moral } \\
\text { attitudes and } \\
\text { values }\end{array}$ & $\begin{array}{l}\text { Des48. Being honest, responsible, respectful to others, and truthful. Avoiding bad practices } \\
\text { (plagiarism, etc.). }\end{array}$ \\
\cline { 2 - 2 } $\begin{array}{l}\text { Ses49. Working for one's own good and the common good, contributing to advancing towards a } \\
\text { fairer and more equitable society. }\end{array}$ \\
\hline $\begin{array}{c}\text { S20. Respecting } \\
\text { ethical and deon- } \\
\text { tological codes }\end{array}$ & $\begin{array}{l}\text { Des50. Integrating ethical and deontological codes into professional activity: respecting human } \\
\text { rights, working thoroughly, respecting professional secrecy, not abusing one's position if in a } \\
\text { position of power, etc. }\end{array}$ \\
\hline
\end{tabular}

Source: Own elaboration.

\section{2.Method}

\subsection{Objectives}

The aim of this work was to test the competence model developed by the research team against the views of four groups of key informants involved in the process. We set out to validate the model with contributions from four groups (stakeholders) regarded as having an interest in the Bologna process (employers, professionals, teachers, and students). 
It was especially important to receive feedback from four of the main groups of people interested in the education provided at university, which are clearly listed in significant documents from the Bologna process of convergence, specifically in regards to tuning (González \& Wagenaar, 2006) and in the European Qualifications Framework (Grupo de trabajo de Bolonia sobre marcos de cualificaciones, 2005). These four groups are teachers and students, as agents involved in the educational process, and employers and professionals, as relevant agents from the field of employment. Employers are relevant as they are responsible for selecting and hiring graduates and are aware of the training they need to enter the world of work, and professionals are significant because they have experience of the world of work and its requirements.

\subsection{Design}

The research project that this work is part of has various objectives and so uses a mixed-method design, including quantitative and qualitative methods (Brannen, 2017; Creswell, 2010). However, the present work is limited to the qualitative part of the design, using focus groups to collect relevant information (Merriam \& Grenier, 2019).

We concentrate on studying the perceptions of key informants involved in the process: employers, university teachers, students in the later stages of bachelor's degrees, and professionals, to test the theoretical model we have developed.
Three discussion groups were held for each of the groups listed from the three branches of knowledge in which the research was performed: Educational Sciences (Universidad Católica de Valencia), Health Sciences (Universidad de Valencia), and Engineering (Universidad Politécnica de Valencia), giving a total of twelve groups.

\subsection{Sample}

The sample was chosen using selective sampling, based on the following criteria:

- Belonging to the three branches of knowledge involved.

- The type and quality of key informants:

- Employers and/or heads of human resources departments in companies (linked to education, health sciences, and engineering).

- Professionals with an interest in the subject (from the three branches).

- University teachers from the three branches of knowledge who have an awareness of the topic and over 20 years' experience.

- Students in the later years of bachelor's degrees with good academic grades (an average of at least 8.5 out of 10).

- Willingness to participate in the study.

The anticipated sample, 4-6 subjects per group, took shape as shown in Table 6. 
TABLE 6. Participants.

\begin{tabular}{|l|c|c|c|c|c|c|c|c|c|c|}
\hline & \multicolumn{3}{|c|}{$\begin{array}{c}\text { Universidad } \\
\text { de Valencia } \\
\text { (Health Sciences) }\end{array}$} & \multicolumn{2}{c|}{$\begin{array}{c}\text { Universidad } \\
\text { Politécnica de Valencia } \\
\text { (Engineering) }\end{array}$} & \multicolumn{2}{c|}{$\begin{array}{c}\text { Universidad Católica } \\
\text { de Valencia } \\
\text { (Educational Sciences) }\end{array}$} & Total \\
\hline & Total & Male & Female & Total & Male & Female & Total & Male & Female & \\
\hline Employers* & 2 & 0 & 2 & 8 & 2 & 6 & 4 & 0 & 4 & 14 \\
\hline Professionals & 6 & 2 & 4 & 7 & 4 & 3 & 5 & 1 & 4 & 18 \\
\hline Teachers & 6 & 3 & 3 & 8 & 4 & 4 & 5 & 0 & 5 & 19 \\
\hline Students & 6 & 4 & 2 & 6 & 4 & 2 & 4 & 1 & 3 & 16 \\
\hline Total & 20 & & & 29 & & & 18 & & & 67 \\
\hline
\end{tabular}

* Four employers were invited but only two could attend the working meeting, both women. Source: Own elaboration.

\subsection{Instruments}

A protocol for the discussion groups was developed with a single question preceded by the following statement:

All people and professionals need to continue learning to improve, adapt, and carry out their duties effectively in a changing socio-economic and cultural setting. As a result, the European Union proposes learning to learn as one of the basic competences students should acquire in the educational system.

In your view, and in relation to learning to learn, given this need to continue learning, what qualities, capacities, skills, etc., must a person acquire or perfect to become a competent and responsible professional when studying for a bachelor's degree at university?

This open approach was preferred over other possibilities such as presenting the research team's model to the participants for valuation. The data collected were rich and suitable for processing.

\subsection{Procedure}

Based on the literature review, the research team prepared a theoretical model of the competence (section 1.3), which, after being subjected to expert evaluation and reviewed, took the form shown in the tables.

The discussion groups were then held at the three universities, with the interventions being recorded. The transcripts were entered into the Atlas.ti.8 software as primary documents, creating a hermeneutic unit without automating the analysis process (Wolf \& Silver, 2018).

The material collected was used for analysing the results and categories were established. This task was done by four trained assessors who were members of the research team and who paid special attention to the process of triangulating sources (Denzin \& Lincoln, 2004; Wolf \& Silver, 2018).

The data processing had two interdependent stages. First, the raw data were worked on at a textual level, identifying segments to be coded in accordance with a list of codes relating to the pre-established analysis categories. The codes were then 
linked at a conceptual level based on the discursive elements and on the theoretical model, creating a map of relationships between elements to corroborate, contest, and complement the initial theoretical model. All of this made it possible to create semantic networks, using the dimensions predicted in the model as the central categories or families: cognitive (D1), metacognitive (D2), affective-motivational (D3), social-relational (D4), and ethical (D5). The model's subdimensions were used to establish the secondary nodes, creating groups with codes labelled «S1», «S2», etc., and their respective titles (Graph 2) ${ }^{2}$. To establish the tertiary nodes, the descriptions/components of these subdimensions were used with codes labelled «Des1», «Des2», etc.

\section{Results}

The presentation of the results first considers the relationships within each dimension to analyse their intradimensional coherence, and then relationships between dimensions that the participants established in order to analyse the model's interdimensional coherence of the model.

Graph 2 presents the results from the 12 groups with the model's five dimensions and subdimensions and with the groundedness scores (g), which indicate how often a comment by participants that is consistent with the corresponding description appears in the text, and the density (d) scores, which refer to how often participants establish relationships between the corresponding description and others mentioned in the group, in each case. Both graphs are important. The dimensions and subdimensions are theoretical elaborations of the model that is being tested, while the comments relating to their descriptions/components - listed in the graph with the title «Des» — are contributions by the participants in the groups and are linked to the theoretical model's variables.

Testimonies by the participants are interspersed in the presentation of the results, according to the primary document to which they refer: PD1, PD2, and PD3 (students), PD4, PD5, and PD6 (employers), PD7, PD8, and PD9 (professionals), and PD10, PD11, and PD12 (teachers). In these literal testimonies, the identifier allocated by Atlas. ti. 8 is given after the primary document, preceded by a colon (:).

\subsection{Intradimensional coherence}

The cognitive dimension (D1) was mentioned most often $(g=98)$. Subdimension S1 («managing information effectively») was mentioned 31 times. This was followed by S2 («communication skills», g = 35) (S2.1, «oral communication skills», $\mathrm{g}=24 ; \mathrm{S} 2.2$, «written communication skills», g = 8; S2.3, «knowledge of foreign languages», $\mathrm{g}=3$ ). There was no mention of S2.3 («knowledge and use of non-verbal language»), while S3 («digital technology») appeared 4 times and S4 («critical and creative thinking») 28 times. Furthermore, D1 had the most internal connections $(d=12)$. This could be because it is one of the most consolidated dimensions, having been among the first to appear 
in research into learning. By calculating their level of internal relationships based on qualitative analysis, the number of connections the participants established between the cognitive subdimensions was relativized, giving a value of $.583^{3}$. «Effective oral communication» (Des3) and «creative personal proposals» (Des13) were the descriptions/components that made the greatest contribution to the internal connections of this dimension, although their intradimensional densities, which illustrate the relationship the participants establish between the components, were not especially high ( $d=2$, in both cases). The second of them also displayed notable groundedness values ( $\mathrm{g}=10$ ), compared with the other cognitive components. They were only exceeded by «searching for, selecting, and processing information effectively» $(\mathrm{g}=19)$.

The metacognitive dimension (D2) had the second highest groundedness value $(\mathrm{g}=76)$. «Questioning learning objectives and setting your own objectives» (Des19) was the idée-force that had the most connections between its components, with 3 associations within the dimension: namely with «knowing your own skills and limitations» (Des17), «analysing, evaluating ...» (Des23), and "problem solving ..." (Des25). Furthermore, this descriptions/component (Des19) and «knowing skills and limitations ..." (Des17) (components from subdimension S6), were mentioned most often by the groups ( $\mathrm{g}=11$ and $\mathrm{g}=10$ respectively). The professors made some interesting testimonies that illustrate these connections: students have to stop being passive and become active and build their own learning. If you are going to build ... you have to ... apply ... the theoretical content you are seeing to the real world. By solving real problems, we introduce ... students to solving a problem that ... you present to them today, and today they might get it wrong because they are at university, but tomorrow ... they might not be able to do it (PD11:3).

The subdimension mentioned most often was S6, from knowledge of oneself ( $\mathrm{g}=$ 35 ), but it should be noted that of all of the descriptions/components of dimension D2, Des25 «solving problems» had the greatest groundedness $(g=26)$, exceeding any other description from this subdimension.

The affective-motivational dimension (D3) appeared 64 times in the comments. The idea mentioned the most was «motivation and curiosity about learning» (description/component Des26) ( $\mathrm{g}=26)$. This idea, from subdimension S10, also contributed more than any other to the internal connection $(d=3)$, being related to «internal attribution» (Des30), «accurate self-image and awareness» (Des31) and «self-efficacy» (Des32). Other descriptions/components were mentioned, also, at a secondary level of importance, "tolerating frustration ...» (Des29) and "accurate self-image and awareness ...» (Des31) ( $\mathrm{g}=$ 14 and $g=11$ respectively). In the words of the employers, students «... are not able to value everything they know and all of their potential» $(\mathrm{PD} 6: 10-11)$.

Participants mentioned the social/ relational dimension (D4) 66 times in 
their testimonies. This dimension had a relative value of .833 , which is the highest relative internal connection. It is not among the longest-established dimensions in the literature, with many of the descriptions/components that comprise it being advances in social learning from the 1990s. Our team deliberately expanded the content of this dimension, using contributions from Bandura's theory of social learning and also including the results of later studies that consider cooperation, working in groups, and other similar aspects. The internal relationship between dimensions might have some relationship with their consolidation in studies published since the last century.

The subdimension with greatest groundedness was S16, attitudes of cooperation and solidarity $(\mathrm{g}=27)$. Within this, the «social skills» description/component was mentioned most, $g=18$. This was followed by S17, «team work» $(\mathrm{g}=23)$, and, finally $\mathrm{S} 15$, «social values» $(\mathrm{g}=16)$.

In this dimension, the description/component Des42, "working in a team» had the highest density of connections ( $d=$ 4), being linked to «valuing interpersonal relationships» (Des37), «cooperating effectively with classmates» (Des39), «social skills» (Des41), and «working contributing ideas ...» (Des43). Two more descriptions/ components had two connections each: «Cooperating with classmates» (Des39), which was linked to Des42 and Des43, and «working contributing ideas, giving and asking for help ..." (Des43), which was linked to Des39 and Des42. The students said that:
Learning how to work in a team is another very important thing because we are very individualistic and ... because you want ... to reach the objective, you skip stages and you do not wait for others who are working more calmly, more quietly, to get there. Every student will have their time, their process (PD1:38).

«Working in a team» (Des42) had a groundedness of 17 , but «social skills» (Des41) were mentioned on 18 occasions. This is interesting because «social skills» did not contribute to the internal connections as much as other subdimensions, with this component only having a density of 3 within this dimension. "Valuing interpersonal relationships ..." also had a significant presence among the participants $(\mathrm{g}=13)$.

The ethics dimension (D5) was mentioned less often. Even so, there were 52 references to it $(\mathrm{g}=52)$, which gives an idea of its importance for participants.

The subdimension with the highest groundedness was S20, «civic and moral attitudes and values» $(\mathrm{g}=26)$, followed by $\mathrm{S} 19$, «responsibility in learning» $(\mathrm{g}=18)$, and S21, «respecting ethical and deontological codes» $(\mathrm{g}=8)$. The descriptions that received the most comments were «being honest, responsible, and respectful to others» (Des48) and «working for one's own good and the common good» (Des49), both of which are components of S20 $(\mathrm{g}=13)$.

This dimension is the one with the fewest internal connections. Des50 «ethical and deontological codes» $(\mathrm{d}=2)$ was 
linked to Des48 ( «being honest, responsible ...») and to Des49 ("working for one's own good and the common good»). The employers said that:

Tolerance in a professional ... is really important. ... We are not talking about justice, but equality. ... another concept I think ... they have not covered ... is the topic of the integrity ... of a person and ... of leaders, essentially (PD4:19-20-21).

\subsection{Coherence between dimensions}

The external relationships between dimensions support the model's consistency, presenting a consistent framework.

The cognitive dimension (D1) related to the metacognitive one (D2): «finding, selecting, and managing information effectively» interacted with «reflection on what to learn, how and what for» and with «analysing, evaluating, and monitoring to improve performance». The teachers said that:

we have to teach them to filter things, ... to know reliable ... sources ... that they know themselves and then have ... a critical vision .... They have to have the method and order, and the steps they have to follow, so they can be independent and solve this problem (PD12:8).

The cognitive dimension was also linked to the affective one: «effective oral communication» was associated with «internal attribution», with the teachers believing that «we have to encourage ... everyone to carry on working on this and feeling confident» (PD6:33).

The metacognitive dimension (D2) was the most connected dimension at the external level. In the participants' comments, «solving problems» (metacognitive component) was associated with «analysis, inference ...» (Des14) and with «questioning underlying assumptions» (Des15) (descriptions/cognitive components). The professionals stated that:

they only concentrate on applying techniques ... they lack ... skills when practising self-criticism or for finding a solution to a particular problem. It is like they constantly need a supervisor ... they do not have that independence when working (PD8: 6-7).

«Knowing your own skills and limitations» (cognitive component) was associated with "tolerating frustration ...» and with an "accurate self-image and awareness» (affective components). The employers said: «it is important how they handle failure ... because when they encounter it ... they freeze» (PD7:22). The students said:

one important ability is to know yourself, because everyone has ... a way of studying ... knowing yourself helps ... to optimise the time ... and then, in the future it also helps you acquire new knowledge (PD1:21).

The social/relational dimension (D4) was associated with the cognitive one (D2): «Valuing interpersonal relationships and working with other people ...» (social component) was related to "prioritising, hierarchising, and organising activities», and with «analysing, evaluating, and monitoring ..." (both metacognitive components). In the words of the employers: 
they have to know how to learn to manage their time ... to be people with empathy ... every person is a different world and we have to know this world to ... get strategies from them so that they continue to learn and can confront different situations in different contexts ... to guide them and make sure they are willing to put into practice the strategies they need, but starting from where they are and how they are (PD6:4).

The affective dimension (D3) was related to the social/relational one (D4): «motivation and curiosity about learning» (affective) with «team work» (social) and «internal attribution» (affective) with «working contributing ideas, giving and asking for help ...» (social):

if you are working in a group to try to learn more on a topic ... if you do not know yourself and what you can offer the group and what the group can offer you ... you cannot embrace all of the possibilities it offers you because ... if you do not know yourself enough to keep moving forwards, you will be limited (PD2:15).

Finally, the social/relational dimension (D4) was related to the ethical one (D5): «cooperating with classmates» (social) with «making an effort to do a good job» (ethical).

It was noted that it is necessary to «take responsibility for your errors, as there is a ... tendency to blame others .... That is one of the major failings when working as a group» (PD6:26).

«Social skills» (social) were also related to being «honest, responsible, and respectful» and with «ethical and deontological codes ...» (ethics).

Table 7 summarises the external relationships in the groups and makes it possible to identify the subdimensions linked to a given dimension and the subdimensions with the most external relationships. The metacognitive dimension has the most external relationships (8), followed by the social (7), cognitive (4), affective, and ethical dimensions, with the last two having the same number of relationships (3).

TABLE 7. External relationships (codified summary).

\begin{tabular}{|c|c|c|}
\hline Dimension & $\mathbf{S}$ & $\begin{array}{l}\text { External } \\
\text { relationships }\end{array}$ \\
\hline \multirow[t]{3}{*}{ Cognitive } & S1 & S6, S8 \\
\hline & $\mathrm{S} 2.1$ & S11 \\
\hline & $\mathrm{S} 4$ & S9 \\
\hline \multirow[t]{4}{*}{ Metacognitive } & S9 & $\mathrm{S} 4$ \\
\hline & $\mathrm{S} 6$ & $\mathrm{~S} 1, \mathrm{~S} 10, \mathrm{~S} 12$ \\
\hline & S8 & S1, S7, S15 \\
\hline & S7 & S15 \\
\hline \multirow{2}{*}{$\begin{array}{l}\text { Affective- } \\
\text { Motivational }\end{array}$} & S10 & S17 \\
\hline & $\mathrm{S} 11$ & $\mathrm{~S} 2.1, \mathrm{~S} 17$ \\
\hline \multirow{3}{*}{$\begin{array}{l}\text { Social/ } \\
\text { Relational }\end{array}$} & S16 & S19 \\
\hline & S15 & S7, S8 \\
\hline & S17 & $\begin{array}{l}\text { S10, S11, S20, } \\
\text { S21 }\end{array}$ \\
\hline \multirow[t]{3}{*}{ Ethical } & S19 & S16 \\
\hline & S20 & S17 \\
\hline & S21 & S17 \\
\hline
\end{tabular}


GRAPHIC 2. Results of processing.

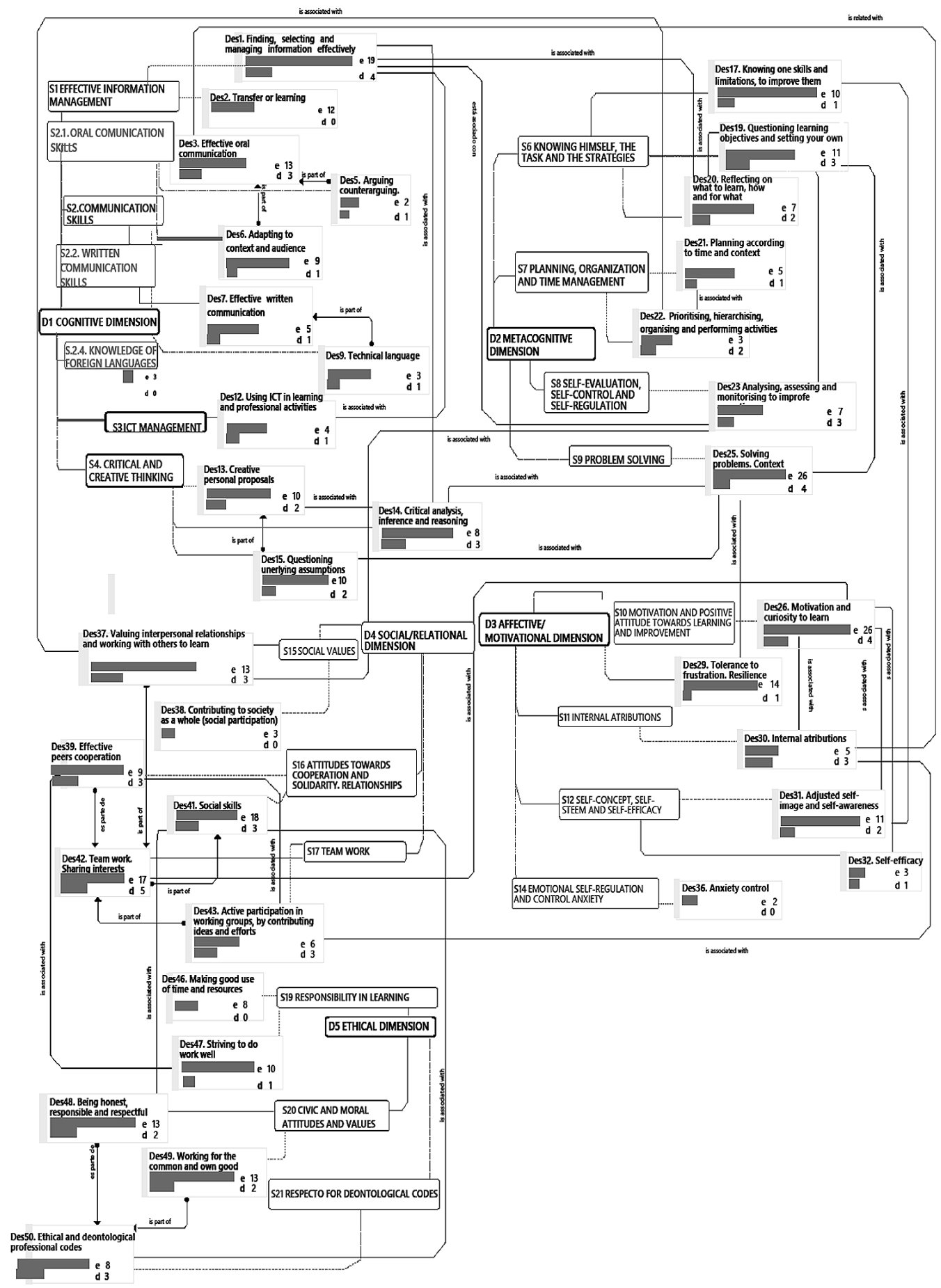

Source: Own elaboration. 


\section{Discussion and Conclusions}

The objective of this work was to compare the LTL competence model developed by the research team with its evaluation by four groups of key informants classed as stakeholders in the Bologna process. Having analysed the data, we can state that the proposed dimensions were endorsed by the participants as were almost all of the subdimensions.

In our opinion, this is very highly significant. On the one hand, the fact that employers, professionals, teachers, and students interpret learning to learn as including the core aspects/dimensions included in the research team's model, without having received any instructions other than the open question formulated for the groups, is an important endorsement of the model's validity. On the other hand, the fact that teachers and students alike, who share in the teaching-learning process, regard its components as important could encourage the inclusion of these components in educational proposals to be implemented in curricula and university degrees. If this is also the case, as indeed it is, with employers and professionals, who know which skills, attitudes, etc., linked to learning to learn are necessary for work and employability, then there are more than enough arguments to opt strongly for teaching this competence in university training.

As we stated above, all of the dimensions were clearly present in the participants' interventions, although not all of the subdimensions were: two of these did not appear in the transcripts (S12, «phys- ical and emotional well-being», and S17, "controlling environmental conditions»). The participants provided a new one: «knowledge of foreign languages» (S2.4). The final decision about whether or not to maintain these subdimensions in the model will be taken based on their statistical validity.

The five subdimensions of the cognitive dimension appear clearly. Subdimension S1, «managing information effectively» ( $\mathrm{g}=31$ ), relating to searching for, selecting, and processing information, is very relevant. It also displays connections with others from the cognitive, metacognitive, and social dimensions, which is important. Another important one is S4, «critical and creative thinking» $(\mathrm{g}=28)$, which is connected to other cognitive, metacognitive, and social dimensions, thus showing its importance and connectedness. This is also true for subdimension S2.1, «oral communication skills» $(\mathrm{g}=24)$, and with less weight, S2.2, «written communication skills» $(\mathrm{g}=8)$ as well as S4, «using digital technologies» $(\mathrm{g}=4)$. We have already mentioned that the participants included a new subdimension, «knowledge of languages» $(\mathrm{g}=3)$. Within $\mathrm{S} 2$, «communication skills", they did not mention S2.3, «knowledge and use of non-verbal language».

The metacognitive dimension also features clear references to its four subdimensions, with S6, «knowledge of oneself, of the task, and of strategies for tackling it, being especially relevant» $(\mathrm{g}=35)$, as it is connected to others from the cognitive and metacognitive dimension. 59 , «problem 
solving», also displays high groundedness $(g=26)$, being related to cognitive, metacognitive, and affective-motivational codes. The other two subdimensions, with lower groundedness, are S7, «planning, organising, and managing time» $(\mathrm{g}=8)$, and S8, «self-evaluation, control, selfregulation».

Within the affective-motivational dimension, four of the five subdimensions are mentioned. Subdimension S10, «motivation and positive attitude towards learning and improvement», is especially relevant, and is connected to other affective and social/relational ones. Also S12, «self-concept, selfesteem, self-efficacy» $(\mathrm{d}=14)$, which is linked to the metacognitive and affective dimension. Lower frequencies are displayed by subdimensions S11, «internal attribution» $(d=5)$, which is linked to others in the affective and relational dimension, and S14, «emotional self-regulation and controlling anxiety» $(d=5)$.

In the social/relational dimension, three of the four subdimensions are mentioned, all of which are important, given their groundedness and density. Subdimension S16, «attitudes of cooperation and solidarity", is mentioned most ( $\mathrm{g}=$ 27), followed by S17, «team work» ( $\mathrm{g}=$ $23)$, and S15, "social values» $(\mathrm{g}=16)$. They also display connections with other social/relational, cognitive, metacognitive, and affective subdimensions.

In the ethical dimension, the three subdimensions are relevant: S20, «civic and moral attitudes and values», is mentioned the most $(\mathrm{g}=26)$, followed by S19, «responsibility in learning» $(\mathrm{g}=18)$, and S21, «respecting ethical and deontological codes» $(\mathrm{g}=8)$. The first two interact with other subdimensions in the social dimension and all three with others from the ethical dimension itself.

\subsection{A consistent model}

The external and internal relationships described reflect a consistent model.

The external relationships show that the initial model is consistent as the dimensions are all connected to each other. It is true that the ethical dimension is the least well-connected, both externally and internally. This is probably not a case of ethical aspects being irrelevant but rather a lack of awareness among the participants. Furthermore, this dimension is not considered by previous theoretical models of the LTL competence (Hautamäki et al., 2002; Hoskins \& Friedriksson, 2008; Stringher, 2014) as it is a new contribution by our group.

The internal relationships that the participants in the groups establish in the cognitive dimension (D1) have a clear logic: «arguing and counterarguing», and «adapting to the setting and audience» form part of "effective oral communication». "Questioning underlying assumptions» and carrying out «critical analysis, inference, and reasoning» are related to the development of "creative personal proposals».

In the case of metacognitive elements (D2), the connection is very consistent: «questioning learning objectives and 
setting your own objectives» is related to «reflection on what to learn, how and what for», «analysing, evaluating, and monitoring to improve performance», and «solving problems».

In the affective/motivational dimension (D3), «motivation and curiosity about learning» was associated with affective elements and those relating to the locus of control, such as «internal attribution", «self-efficacy», and having an «accurate self-image and awareness».

A similar thing happened with the ethics dimension (D5): following «ethical and deontological codes ...» was linked to being "honest, responsible, and respectful» and to «working for one's own good and the common good", as these codes generally specify in any professional sphere or area of knowledge.

The most complex internal connections are those from the social/relational dimension (D4). As the subdimensions are so connected in relative terms, it is hard to establish groupings according to the links. We have interpreted this as an indicator of the internal coherence of this dimension given that the participants related all of the subdimensions with each other, except for one: contribute to society as a whole.

The dimensions with the greatest relative weight of internal connections after the social/relational one were the cognitive and metacognitive ones (Graph 3).

GRAPH 3. Relative internal connection weights.

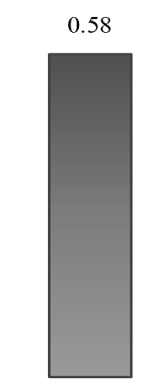

Cognitive

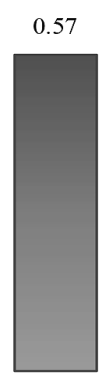

Metacognitive

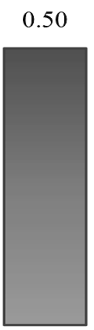

Affective-Motivational
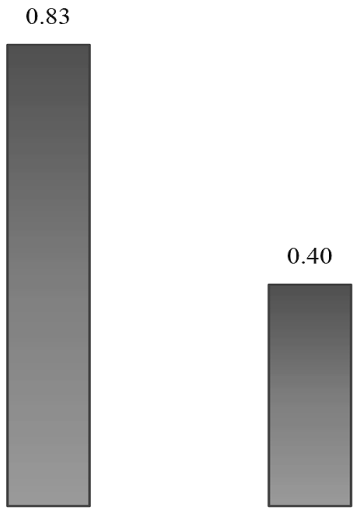

Social-Relational

\subsection{Connections between dimensions}

Aside from the groundedness and density of each subdimension, the intradimensional connection is an indicator of delineation.
The participants attributed an important relative internal connection to the dimensions that appeared earliest in the scientific literature. So, the cognitive dimension had the highest relative 
intradimensional score, followed by the metacognitive dimension, the affective-motivational one, and the ethical one. Nonetheless, the social/relational dimension was the most delineated one in accordance with its internal connection.

In the literature, the first three appear early and their content remains fairly stable, with new contributions based on research being absorbed (Panadero, 2017; Thoutenhoofd \& Pirrie, 2013). The first strategic learning models include three major types of strategy: information processing (cognitive), support (affective-emotional), and metacognitive (Danserau, 1985; Weinstein \& Mayer, 1985; Weinstein, 1987).

The same happens with self-regulated learning models. Zimmerman's first works, based on Bandura's theory of social cognition, featured aspects of the setting, behaviour, and person in their tripartite model, always from the perspective of the learner who self-regulates in the three fields. Later on, in his cyclical model from 2000, he defined three phases, which are maintained in the 2009 and 2013 models (Zimmerman, 2000, 2013; Zimmerman \& Moylan, 2009): the forethought phase (including analysis of tasks and self-motivation), the performance phase (self-observation and self-control), and the self-reflection phase (self-judgement and self-reaction). The cognitive, metacognitive, and affective-emotional dimensions clearly appear in them. Boekaerts (1996) also includes them in her model.

It is true that in the research that sets out the strategic learning construct, the learner is above all seen as an individual subject who confronts learning, and to some extent as an «isolated learner», who must be able to manage his or her learning processes autonomously. Nonetheless, in the theory of self-regulated learning, more importance is increasingly being given to constructing knowledge in the community, in line with shared and situated cognition, along with others, in context, and with a socialised and cooperative metacognitive approach (Thoutenhoofd \& Pirrie, 2013) so that each individual builds his or her own metacognitive and self-regulation - coregulation - skills helped by others (Allal, 2019). This is the case because of the social-cognitive foundations of this construct, which emphasises interaction between people, skills, context, and their situation (Panadero, 2017).

It is also true that the studies that have acted as a reference point for the social/dimensional dimension do not follow a clear continuous pattern over time, which conversely is common in all of the other dimensions apart from the ethical one, which does not have precedents. Bandura's theory of cognitive-social learning (1986) and the social-cognitive focus in general inspired the design of our theoretical model along with other studies that opened up new lines in the social dimension of learning, without necessarily advancing on the basis of earlier foundations. For example, the works of Kagan (1990), which show the relevance of aspects such as cooperation and interdependence in the learning process, or those of Shaver (1991), referring to the acquisition and reformulation of 
social values. These aspects were also introduced in the initial theoretical model, making it clear that the social/relational dimension has no clear starting point in the history of the research. There are various starting points, and moves forward have not always been based on what came before.

The ethical dimension is a contribution by this research team, as stated above, and is clearly validated by the participants in the groups, who mentioned aspects included in the dimension 52 times, something that is highly relevant. No model of the LTL competence can neglect the ethical aspects relating to learning. Surprisingly, the models of the learning to learn competence currently available do not explicitly contemplate this dimension, despite the steady incorporation into university education of models such as service learning, which have a clear ethical element (González-Geraldo, Jover, \& Martínez, 2017), works that consider how to include ethical questions in learning processes (De Reuyter \& Schinkel, 2017), and works that refer to the relationship between active citizenship, its ethical aspects, and learning to learn (Hoskins \& Deakin Crick, 2010). However, we should note that the most recent formulation of the competence developed by the EC (2018) does refer to ethical elements: it alludes to the need to develop collaborative and tolerant attitudes and to feel empathy, respect others, understand different points of view, overcome prejudices, and compromise, acting with integrity. Nonetheless, these elements appear to be linked to social aspects of learning, rather than being something with their own dimension.

In our view, giving social-relational aspects the importance they deserve, along with cognitive, metacognitive, and affectivemotivational ones, and including the ethical dimension in the competence's essential dimensions makes a significant contribution to enriching it and permits a comprehensive model that can serve as a reference point for work in educating students, both at university and in other contexts.

\subsection{Limitations and future lines of work}

It is surprising that some subdimensions have not appeared as strongly as we expected. «Controlling anxiety» (affectivemotivational dimension) and «arguing and counterarguing» (cognitive dimension) obtained $\mathrm{g}=2$, for example. This low level of groundedness could derive from the participants' lack of awareness. It could also be from immediacy, as in discussion groups participants have to answer the questions they are asked without knowing what they will be in advance and without time to reflect on them. This could also relate to how the primary documents are interpreted. This is one of the principal limitations of the use of Atlas.ti.8.

The software used does not automatically interpret and analyse the data; the team of researchers has to do this. To overcome the problems of subjectivity, various members of the team performed the analysis and interpretation with the texts, without limiting themselves to carrying out mechanical operations and deliberating on potential coding errors. 
It would be useful to complement the results of this study with a comparison of the perspectives of the four audiences involved. The future of this research involves validating the content of the competence with reliability tests and tests for differences between subjects. On this basis, an evaluation questionnaire will then be designed that collects the competence's content in order to evaluate it, thus making it possible to collect information and make curriculum proposals for how to teach this competence.

\section{Notes \\ ${ }^{1}$ "Operational design of the "learning to learn" com. petence for university degrees, evaluation instruments and teaching proposals». Research funded by Spain's Ministry of Economy, Industry, and Competitiveness. Code EDU2017-83284-R. Call for applications for fun. ding for R\&D\&i projects Retos de la Sociedad, 2017.}

${ }^{2}$ Of the twenty subdimensions presents in the model, only two do not appear in the graph: controlling en. vironmental conditions (social/relational dimension) and physical and emotional well-being (affective-moti. vational dimension). The participants did not mention them.

3 The formula used for the calculation is $n / N$, where $\mathrm{n}$ is the number of connections or relationships of a dimension and $\mathrm{N}$ is the total number of components of the dimension. This makes it possible to obtain a relative score: in this case, there are seven internal connections and twelve components. $7 / 12=.583$.

\section{References}

Allal, L. (2011). Pedagogy, didactics and the coregulation of learning: a perspective from the French-language world of educational research. Research Papers in Education, 26 (3), 329-336.

Bandura. A. (1986). Social Foundations of Thought and Action: A Social Cognitive Theory. Englewood Cliffs, NJ: Prentice-Hall.
Boekaerts, M. (1996). Self-regulated learning at the junction of cognition and motivation. European Psychologist, 1 (2), 100-112.

Brannen, J. (2017). Mixing methods: qualitative and quantitative research. London: Routledge.

Caena, F. (2019). Developing a European Framework for the Personal, Social \& Learning lo Learn Key Competence. Luxemboug: Publications Office of the European Union. Retrieved from https:// bit.ly/2vBzK8A (Consulted on 2019-10-10).

Cortina, A. (2013). ¿Para qué sirve realmente la ética? Barcelona: Paidós.

Creswell, J. W. (2010). Mapping the developing landscape of mixed methods research. In Handbook of mixed methods in social and behavioral research. Second Edition (pp. 45-68). Thousand Oaks, CA: Sage.

Danserau, D. F. (1985). Learning Strategy Research. In H. F. O’Neil (Ed.), Learning Strategies (pp. 209-240). New York: Academic Press.

De Reuyter, D., \& Schinkel, A. (2017). Ethics education at the university: from teaching an ethics module to education for the good life. Bordón. Revista de Pedagogía, 69 (4), 125-138. doi: https://doi.org/10.13042/Bordon.2017.690409

Deakin Crick, R., Stringher, C., \& Ren, K. (2014). Learning to Learn: International perspectives for theory and practice. London: Routledge.

Denzin, N., \& Lincoln, Y. S. (Eds.) (2006). The Sage Handbook of Qualitative Research. Thousand Oaks, CA: Sage.

EI-ESU (2012). Bologna with student eyes 2012. Retrieved from https:/www.esu-online.org/ wp-content/uploads/2016/07/BWSE2012-online1.pdf (Consulted on 2019-10-06).

European Commission (EC) (2006). Recomendación del Parlamento Europeo y del Consejo de 18 de diciembre de 2006 sobre competencias clave para el aprendizaje permanente. Retrieved from https:// bit.ly/2QNgYmo (Consulted on 2020-03-26).

European Commission (EC) (2018). Anexo de la Propuesta de Recomendación del Consejo relativa a las competencias clave para el aprendizaje permanente. Retrieved from https://g00.gl/YD9pDw (Consulted on 2019-10-06).

Flavell, J. H., \& Wellman, H. M. (1977). Metamemory. In R. V. Kail Jr. \& J. W. Hagen (Eds.), Perspectives on the development of memory and cognition (pp. 3-33). Hillsdale: LEA. 
Gargallo López, B., Pérez-Pérez, C., García-García, F. J., Giménez Beut, J. A., \& Portillo Poblador, N. (2020). La competencia aprender a aprender en la universidad: propuesta de modelo teórico. Educación XX1, 23 (1), 19-44, doi: https://doi. org/10.5944/educxx1.23367

González, J., \& Wagenaar, R. (2006). Tuning Educational Structures in Europe II. La contribución de las universidades al proceso de Bolonia. Bilbao: Universidad de Deusto. Retrieved from http://www.deusto-publicaciones.es/deusto/pdfs/ tuning/tuning04.pdf (Consulted on 2019-10-04). González-Geraldo, J. L., Jover, G., \& Martínez, M. (2017). La ética del aprendizaje servicio en la universidad: una interpretación desde el pragmatismo. Bordón. Revista de Pedagogía, 69 (4), 63-78. doi: https://doi.org/10.13042/ Bordon.2017.690405

Grace, S., Innes, Ev., Patton, N., \& Stockhausen, L. (2017). Ethical experiential learning in medical, nursing and allied health education: A narrative view. Nurse Education Today, 51, 23-33.

Grupo de trabajo de Bolonia sobre marcos de cualificaciones (2005). Informe sobre: un marco de cualificaciones para el espacio europeo de educación superior. Retrieved from http://www. unizar.es/eees/doc/Marco_cualificaciones.pdf (Consulted on 2020-03-03).

Hautamäki, J., Arinen, P., Eronen, S., Hautamäki, A., Kupiainen, S., Lindblom, B., Scheinin, P. (2002). Assessing Learning-to-Learn: A Framework. Helsinki: Centre for Educational Assessment, Helsinki University/National Board of Education. Hautamäki, J., \& Kupiainen, S. (2014). Leaning to learn in Finland: Theory and policy, research and practice. In R. Deakin Crick, C. Stringher, \& K. Ren (Eds.), Learning to learn (pp. 179200). London and New York: Routledge.

Hoskins, B., \& Deakin Crick, R. (2010). Competences for learning to learn and active citizenship: different currencies or two sides of the same coin? European Journal of Education, 45 (1). doi: https://doi.org/10.1111/j.1465-3435.2009.01419.x

Hoskins, B., \& Fredriksson, U. (2008). Learning to learn: what is it and can it be measured. Ispra: European Commission. Joint Research Centre, Institute for the Protection and Security of the Citizen. Centre for Research on Lifelong Learning (CRELL).
Jornet Meliá, J. J., García-Bellido, R., \& González-Such, J. (2012). Evaluar la competencia aprender a aprender: una propuesta metodológica. Profesorado. Revista de currículum y formación del profesorado, 16 (1), 103-123.

Kagan, S. (1990). Cooperative learning resources for teachers. San Juan de Capistrano, CA: Resources for Teachers.

Kass, M., \& Faden, R. R. (2018). Ethics and learning health care: the essentials roles of engagement, transparency, and accountability. Learning Health Systems, 2 (4), 1-3. doi: https://doi. org/10.1002/lrh2.10066

Kupiainen, S., Hautamäki, J., \& Rantanen, P. (2008). EU pre-pilot on learning to learn: Report on the compiled data, 2008-1190/001001 TRA-TRINDC. Bruselas: Comisión Europea.

Merriam, Sh. B., \& Grenier, R. S. (Eds.) (2019). Qualitative research in practice: examples for discussion and analysis. San Francisco: Jossey-Bass.

Moreno, A., Cercadillo, L., \& Martínez, M. (2008). Learn European Project. Pre-Pilot Study National Report. Madrid: Ministerio de Educación, Cultura y Deporte.

Moreno, A., \& Martín, E. (2014). The Spanish approaching to learning to learn. In R. Deakin Crick, C. Stringher, \& K. Ren, Learning to learn (pp. 196-213). London and New York: Routledge.

Muñoz-San Roque, I., Martín-Alonso, J. F., Prieto-Navarro, L., \& Urosa-Sanz, B. (2016). Autopercepción del nivel de desarrollo de la competencia de aprender a aprender en el contexto universitario: propuesta de un instrumento de evaluación. Revista de Investigación Educativa, 34 (2), 369-383.

Panadero, E. (2017). A Review of Self-Regulated Learning: Six Models and Four Directions for Research. Frontiers in Psychology, 8 (422), 1-28.

Pintrich, P. R. (2004). A conceptual framework for assessing motivation and self-regulated learning in college students. Educational Psychology Review, 16 (4), 385-407.

Shaver, J. P. (Ed.) (1991). Handbook of research on social studies teaching and learning. New York, NY: Maxwell Macmillan International. 
Schmeck, R. R. (1988). Learning strategies and learning styles. Nueva York: Plenum Press.

Stringher, C. (2014). What is learning to learn? A learning to learn process and output model. In R. Deakin Crick, C. Stringher, \& K. Ren (Eds.), Learning to learn (pp. 9-32). London and New York: Routledge.

Thoutenhoofd, E. D., \& Pirrie, A. (2013). From self-regulation to learning to learn: observations on the construction of self and learning. British Educational Research Journal, 41 (1), 72-84.

Villardón-Gallego, L., Yániz, C., Achurra, C., Iraurgi, I., \& Aguilar, M. C. (2013). Learning competence in university: development and structural validation of a scale to measure. Psicodidáctica, 18 (2), 357-374.

Weinstein, C. E. (1987). LASSI User's Manual. Clearwater, FL: H\&H and Publishing Company.

Weinstein, C. E. (1988). Assessment and training of student learning strategies. In R. R. Schmeck (Ed.), Learning strategies and learning styles (pp. 291-316). New York: Plenum Press.

Weinstein, C. E., Husman, J., \& Dierking, D. (2002). Self-Regulation Interventions with a

Wolf, N. H., \& Silver, Ch. (2017). Qualitative analysis using Atlas.ti. New York: Routledge.

Yip, M. C. W. (2012). Learning strategies and self-efficacy as predictors of academic performance: a preliminary study. Quality in $\mathrm{Hi}$ gher Education, 18 (1), 23-34.

Zimmerman, B. J. (2000). Attaining self-regulation: a social cognitive perspective. In M. Boekaerts, P. R. Pintrich, \& M. Zeidner (Eds.), Handbook of Self-Regulation (pp. 13-40). San Diego, CA: Academic Press.

Zimmerman, B. J. (2013). From cognitive modeling to self-regulation: a social cognitive career path. Educational Psychologist, 48, 135-147.
Zimmerman, B. J., \& Moylan, A. R. (2009). Selfregulation: where metacognition and motivation intersect. In J. Hacker, J. Dunlosky, \& A. C. Graesser (Eds.), Handbook of Metacognition in Education (pp. 299-315). New York: Routledge.

\section{Authors' biographies}

Bernardo Gargallo López has a doctorate in Philosophy and Educational Sciences from the Universidad de Valencia where he is Professor of Theory of Education. National First Prize in Educational Research in 2000 and 2002. His current research interests and the competitive projects he has directed focus on teaching and learning at university. He is head of the GIPU-EA research group.

(iD https://orcid.org/0000-0002-2805-4129

Fran J. García-García is a Research Trainee in the Department of Theory of Education, Universidad de Valencia, with a grant for University Teacher Training (FPU) from Spain's Ministry of Education, Culture and Sports (FPU17/00156). Winner of the Special Master's Prize for Special Education. His most recent works have been on university pedagogy and students' learning in higher education.
(iD https://orcid.org/0000-0002-6267-0080

Inmaculada López Francés has a doctorate in Education from the Universidad de Valencia and is an Assistant Professor. Degree and doctoral prizes from the Universidad de Valencia. Her research interests include sexual, affective, and identity diversity, and gender and university teaching.

(iD https://orcid.org/0000-0003-1178-9054 
Miguel Ángel Jiménez Rodríguez has a doctorate in Philosophy and Educational Sciences from the Universidad de Valencia. Special Degree Prize. Associate Professor at the Universidad Católica de Valencia. He has been Vice-Dean for Educational Psychology, Primary School Teaching, and Social Education. Director of the Educa-Acción lifelong learning centre at the same University. Member of the GIPU-EA research group. He has a long research career in the field of competences.

iD https://orcid.org/0000-0002-1550-6157
María Salomé Moreno Navarro is an Architect and University Specialist in University Pedagogy at the Universidad Politécnica de Valencia, where she is a Professor of Practice. She collaborates on competitive projects with the GIPU-EA research group and has participated in two Universidad Politécnica de Valencia Innovation and Improvement projects.

iD https://orcid.org/0000-0003-4578-5959 


\section{revista española de pedagogía año 78, n. ${ }^{\circ}$ 276, mayo-agosto 2020 \\ Spanish Journal of Pedagogy \\ year 78, n. 276, May-August 2020 \\ Table of Contents \\ Sumario}

\section{Editorial}

\section{Editorial}

José Antonio Ibáñez-Martín

An educational consideration on the pandemic:

endure...and progress

Una consideración educativa sobre la pandemia: resistir...

y adelantar

181

\section{Studies}

\section{Estudios}

Bernardo Gargallo López, Fran J. García-García,

Inmaculada López-Francés, Miguel Ángel Jiménez

Rodriguez, \& Salomé Moreno Navarro

The learning to learn competence: An assessment

of a theoretical model

La competencia aprender a aprender: valoración de un modelo teórico

187

Miguel A. Santos Rego, Maria José Ferraces Otero, Ígor Mella Núñez, \& Ana Vázquez-Rodriguez

University, civic-social competences, and the

labour market

Universidad, competencias cívico-sociales y mercado de trabajo
Jesús Miguel Jornet Meliá, María Jesús Perales

Montolío, \& José González-Such

The concept of validity of teaching evaluation processes

El concepto de validez de los procesos de evaluación de la docencia

Fernando Acevedo Calamet

Explanatory factors for dropout from higher education in unfavourable socio-academic contexts

Factores explicativos del abandono de los estudios en la educación superior en contextos socio-académicos desfavorables

\section{Notes \\ Notas}

Maria-Carmen Ricoy, \& Cristina Sánchez-Martinez

A systematic review of tablet use in primary education Revisión sistemática sobre el uso de la tableta en la etapa de educación primaria

Jaime Carcamo-Oyarzun, \& Christian Herrmann Construct validity of the MOBAK test battery for the assessment of basic motor competencies in primary school children

Validez de constructo de la batería MOBAK para la evaluación de las competencias motrices básicas en escolares de 
Concha Iriarte Redin, Sara lbarrola-Garcia, \& Maite Aznárez-Sanado

Proposal for a school mediation evaluation tool (MEQ)

Propuesta de un instrumento de evaluación

de la mediación escolar (CEM)

\section{Bruno Echauri Galván, \& Silvia Garcia Hernández}

Translating in colours: Translation as an

assessment tool for reading comprehension

in English as a second language

Traducir en colores: la traducción como herramienta de evaluación de la comprensión lectora en inglés como lengua extranjera

\section{Book reviews}

Esteban-Bara, F. (2019). La universidad light: Un análisis de nuestra formación universitaria [The light university: An analysis of our university education] (José L. González.

Geraldo). Ruiz-Corbella, M., \& García-Gutiérrez, J. (Eds.) (2019). Aprendizaje-Servicio. Los retos de la evaluación [Service learning: The challenges of evaluation] (Andrea Muñoz Villanueva). Lafforgue, L. (2019). Recuperemos la escuela [Reclaiming the school] (Beatriz Gálvez). FerracesOtero, M. J., Godás-Otero, A., \& García-Álvarez, J. (2019). Cómo realizar un estudio científico en ciencias sociales, de la educación y de la salud [How to do a scientific 327 study in the social, educational, and health sciences (Carolina Rodríguez-Llorente).

This is the English version of the research articles and book reviews published originally in the Spanish printed version of issue 276 of the revista española de pedagogía. The full Spanish version of this issue can also be found on the journal's website http://revistadepedagogia.org.

ISSN: 0034-9461 (Print), 2174-0909 (Online)

https://revistadepedagogia.org/

Depósito legal: M. 6.020 - 1958

INDUSTRIA GRÁFICA ANZOS, S.L. Fuenlabrada - Madrid 